\title{
On Lagrangian approach to self-dual gauge fields in spacetime of nontrivial topology
}

\author{
Igor Bandos \\ $U P V / E H U$, \\ P.O. Box 644, 48080 Bilbao, Spain \\ IKERBASQUE, Basque Foundation for Science, \\ 48011, Bilbao, Spain \\ E-mail: igor.bandos@ehu.es
}

ABSTRACT: We study the Lagrangian description of chiral bosons, p-form gauge fields with (anti-)self-dual gauge field strengths, in $D=2 p+2$ dimensional spacetime of nontrivial topology. We show that the manifestly Lorentz and diffeomorphism invariant PastiSorokin-Tonin (PST) approach is consistent and produces the (anti-)self-duality equation also in topologically nontrivial spacetime. We discuss in what circumstances the nontrivial topology makes difference between two disconnected, da-timelike and da-spacelike branches of the PST system, the gauge fixed version of which are described by not manifestly invariant Henneaux-Teitelboim (HT) and Perry-Schwarz (PS) actions, respectively.

Keywords: Gauge Symmetry, Field Theories in Higher Dimensions, Field Theories in Lower Dimensions, Global Symmetries

ARXIV EPRINT: 1406.5185 


\section{Contents}

1 Introduction 1

$2 \quad$ PST Lagrangian for 6D selfdual gauge fields 4

2.12 -form gauge field in $\mathrm{D}=6 \quad 4$

2.1.1 Free action for 2-form gauge potential in $M^{1+5} \quad 4$

2.1.2 (Anti-)self duality equation 5

2.2 Duality invariant action for the 2-form gauge field. PST, HT and PS actions; $d a$-timelike and $d a$-spacelike branches of PST system 6

2.2.1 PST action 6

$\begin{array}{lll}\text { 2.2.2 Lagrangian equations from PST action } & 7\end{array}$

$\begin{array}{lll}\text { 2.2.3 Gauge symmetries and branches of the PST system } & 7\end{array}$

2.2.4 Gauge fixed form of the PST action: HT and PS actions 8

2.2.5 Anti-self-duality from Lagrangian equations of motion 9

3 PST action and equations of motion in topologically nontrivial $M^{1+5} \quad 10$

3.1 First order form of the PST Lagrangian equations in a topologically non$\begin{array}{ll}\text { trivial spacetime } & 10\end{array}$

3.2 'Semilocal symmetry' of the PST action in topologically nontrivial $M^{6} \quad 12$

$\begin{array}{lll}3.3 & \text { On Noether current for semilocal symmetry } & 14\end{array}$

$\begin{array}{lll}\text { 3.3.1 A speculation on possible alternative } & 16\end{array}$

3.4 (Anti-)self-duality equation from PST action in topologically nontrivial $M^{6} \quad 17$

$\begin{array}{lll}3.5 & \text { Summarizing the case of chiral 2-form gauge potential in } 6 \text { dimensions } & 19\end{array}$

4 Prototype of the topological gauge symmetry in 2D PST action for chiral bosons $\quad \mathbf{2 0}$

4.1 Semi-local symmetry as gauge symmetry of FJ and $d a$-timelike PST actions 22

4.2 Chirality equation as gauge fixed form of the Lagrangian equations of the FJ action and of the da-timelike branch of the PST action 23

4.3 Issues of anti-FJ action and $d a$-spacelike branch of the 2d PST system 23

4.4 A speculation on alternative canonical formalism in 2d 24

$\begin{array}{ll}\text { 4.5 Chiral bosons on a Riemann surface } & 25\end{array}$

5 Twisted anti-self-duality of p-form gauge fields from the PST action in a $\mathrm{D}=\mathbf{2 p}+\mathbf{2}$ dimensional spacetime of nontrivial topology 26

5.1 Twisted anti-self-duality in $D=2 p+2 \quad 26$

5.2 PST action for chiral bosons in $M^{2 p+2} \quad 27$

5.3 Twisted anti-self-duality from PST action in topologically nontrivial $M^{2 p+2} \quad 28$

6 Conclusions $\quad 30$ 


\title{
A On first order form of the Lagrangian equations of the PST system in flat spacetime
}

\author{
B Noether currents and Noether charges in a 6d theory of 2-form gauge \\ potential
}

\section{Introduction}

The PST (Pasti-Sorokin-Tonin) approach [1, 2] provides a manifestly Lorentz invariant Lagrangian description of the self-dual gauge fields as well as of more general dualityinvariant theories. One of the simplest examples is provided by $\mathrm{D}=6$ theory of chiral 2-form potential $B_{2}$, where the PST action allows to reproduce the self-duality or antiself duality condition $H_{3}= \pm * H_{3}$ for the field strength $H_{3}=d B_{2}$. It is also known the Dirac-Born-Infeld type version of this model producing the nonlinear generalization of the self-duality conditions and describing the M-theory 5-brane (M5-brane) in its bosonic limit [3] and in a complete form $[4,5]$.

The PST action involves an auxiliary scalar field $a(x)$ which enters the Lagrangian only through its derivatives $\partial_{\mu} a(x)$. Hence its shift by constant parameter is a (Pecci-Queen) symmetry. But moreover, the PST scalar $a(x)$ is a Stückelberg field as far as the PST action possesses a gauge symmetry (so-called second PST or PST2 symmetry) which can be 'parametrized' by (almost) arbitrary variations $\delta a(x)$ of $a(x)$. The square of derivatives of this PST scalar enters the denominator of the Lagrangian so that it is not allowed to be zero or constant, but it can be gauged to coincide with e.g. one of the space or time coordinates (but not with their light-like combinations $x^{ \pm}=t \pm x$ ). Fixing $d a=d t$ in the PST Lagrangian for 6D chiral bosons we arrive at the non-manifestly Lorentz invariant Henneaux-Teitelboim (HT) action [6]; while fixing, say, $d a=d x^{1}$, we arrive at the PerrySchwarz-type (PS) action [7].

As we will discuss in the main text, the gauges $d a=d t$ and, say, $d a=d x^{1}$ cannot be connected by a nonsingular PST2 gauge transformations. Thus the PST action actually describes a dynamical system with two branches, where, respectively, the gauge $d a=d t$ and the gauge $d a=d x^{1}$ can be fixed; let us call these $d a$-timelike and da-spacelike branches of the PST-system. In topologically trivial situation these branches are classically equivalent in the sense that both can be used to produce the (anti-)self-duality as a gauge fixed version of the equations of motion.

In this paper we study the PST system in spacetime of nontrivial topology. The standard PST action is well defined if the spacetime admits a nowhere vanishing vector field. This is always the case in the spacetime with metric of Lorentzian signature. To be more explicit, generically the nowhere vanishing timelike vector field exists in it; this can be related with the exterior derivative of the PST scalar $d a$ thus allowing for a $d a$ timelike branch of the PST system (and for its gauge fixed version, the HT action), while 
to have also da-spacelike branch (and its gauge fixed version, the PS action), we need the Lorentzian spacetime to admit the second, spacelike nowhere vanishing vector field.

In a topologically nontrivial spacetime with Euclidean metric which does not admit nowhere vanishing vector field, the standard PST construction is not well defined. However, if it allows for existence of a nowhere vanishing 'q-plane field' with $q>1$, then a modified version of the PST action with several $(q)$ PST scalars $[8,9]$ can be constructed. These cases (which are associated with different $D=q+(D-q)$ splittings of spacetime [10-12]) are beyond the scope of this paper.

The standard PST action possesses one more gauge symmetry, the so-called PST1 symmetry, which leaves inert the PST scalar $a(x)$ but plays a very important role in derivation of the self-duality (or duality) equation. The point is that the variation of the PST action produces (a special type of the) second order equation, and the self-duality equation can be deduced from it by using the gauge fixing of the PST1 symmetry. In the original discussion of $[1,2]$, as well as in all presently known applications of PST technique (see e.g. [13-17]), this procedure included the stage of solving the condition for some p-form to be closed, $d G_{p}=0$, by writing it as an exact form, $G_{p}=d P_{p-1}$. Then one can show that $P_{p-1}$ can be gauged away by the PST1 symmetry and that the remaining equation $G_{p}=0$ is equivalent to the self-duality equation for the field strength of the gauge potential.

The procedure described above is literally applicable to the case of flat or topologically trivial spacetime. To be more precise, it is valid when the $p$-th Betti number of the spacetime manifold $M$ vanishes, $b_{p}=\operatorname{dim}\left(\mathbb{H}^{p}(M, \mathbb{R})=0\right.$. However, if $b_{p} \neq 0$, there exists a (set of) closed but not exact $p$-form(s) $\omega_{p}^{\Lambda}, \Lambda=1, \ldots, b_{p}$ so that the general solution of $d G_{p}=0$ reads $G_{p}=\sum_{1}^{b_{p}} c_{\Lambda} \omega_{p}^{\Lambda}+d P_{p-1}$ with constant $c_{\Lambda}$. (This is the place to stress that our discussion here is schematic, and actually in some cases we will arrive at the expressions with functions $l_{\Lambda}(a(x))$ of the PST scalar $a(x)$ instead of constants $\left.c_{\Lambda}\right)$. At the first glance, this seems to spoil the derivation of the self-duality equation from the PST Lagrangian.

The main aim of the present paper is to show that this is not the case: the PST approach is pretty consistent and is able to produce the wanted (anti-)self-duality equations also in the case of topologically nontrivial spacetime.

To see this one has to notice the presence of an unusual type of symmetry parametrized by function(s) of the PST scalar, $f(a(t, \vec{x}))$, which we call "semi-local symmetry". ${ }^{1}$ In the da-timelike branch, where the PST scalar can be gauge fixed to coincide with the time coordinate, this semi-local symmetry is a gauge symmetry which can be used in the above mentioned derivation of the self-duality equation. In contrast, in the da-spacelike branch, where $a(x)$ can be gauge fixed to coincide with one of the special coordinate, the semilocal symmetry is an infinite dimensional local symmetry, similar to the two dimensional conformal symmetry. This cannot be used to fix any gauge and, as a result, in some topologically nontrivial situations the self-duality equation cannot be produced in this branch.

\footnotetext{
${ }^{1}$ When this paper was finished the author have become aware that for the noncovariant HT action in $\mathbb{R} \otimes M^{5}$ spacetime with $b_{2}\left(M^{5}\right) \neq 0$ such a symmetry had been found in [18]. The author thanks Marc Henneaux for having brought this paper to his attention.
} 
Thus, interestingly enough, the nontrivial spacetime topology singles out the $d a$ timelike branch of the PST system.

The rest of this paper is organized as follows. In section 2 we review the PST approach to Lagrangian description of the chiral bosons in flat six dimensional spacetime. We obtain Lagrangian equations of motion form the PST action for two-form gauge potential $B_{2}$, describe the gauge symmetries of this action, the set of which includes the so-called PST1 and PST2 gauge symmetries, and show that these allow to fix a gauge where the Lagrangian equations of motion reduce to the anti-self-duality equation.

In section 3 we elaborate this formalism in topologically nontrivial spacetime $M^{6}=$ $M^{1+5}$ and prove its consistency. In section 3.1. we obtain the first order form of the PST Lagrangian equations and find that in certainly topologically nontrivial spacetimes, including $M^{1+5}$ with $b_{2} \neq 0$, this contain additional topological contribution defined by a number of functions of one variables. In section 3.2. we show that in such spacetimes, in addition to the PST1 and PST2 gauge symmetries, the PST action also possesses an additional semi-local symmetry and that the additional topological contributions to the first order form of the Lagrangian equations can be compensated or generated by transformations of this semi-local symmetry. In section 3.3. we show that in the $d a$-timelike branch of the PST system, as well as in its gauge fixed version described by HT action, the semi-local symmetry is a gauge symmetry, while in the da-spacelike branch of the PST system and in its gauge fixed version described by the PS action, this is an infinite dimensional rigid symmetry (similar to $2 \mathrm{~d}$ conformal symmetry). This allows us to derive (in section 3.4 .) the anti-self-duality equations as gauge fixed version of the Lagrangian equations of motion which follows from the $d a$-timelike PST action and HT action.

In section 4 we discuss the two dimensional PST action for the chiral bosons, where the counterpart of semi-local symmetry is present also in the case of topologically trivial $2 \mathrm{~d}$ spacetime. The case of chiral bosons in a topologically nontrivial spacetime of arbitrary even dimension $D=2 p+2$ is addressed in section 5 . A strong similarity with 6 D case allows us to be short and to restrict ourselves by presenting the basic equations and formulating the results for this general case. We conclude in section 6 .

In appendix A we give additional technical details on derivation of the first order form of the PST Lagrangian equations in flat spacetime. In appendix B we discuss the properties of Noether currents for gauge symmetries on a specific example of 2-form gauge fields theories in $\mathrm{D}=6$.

For the reader convenience we have make the presentation of $\mathrm{D}=6$, of $\mathrm{D}=2$ and of the general $\mathrm{D}=2 \mathrm{p}+2$ cases self-sufficient, so that each of these three can be read separately; the price to pay was repetition of the basic statements, although with some specific modifications.

\section{Abbreviations:}

- PST is used for Pasti-Sorokin-Tonin approach [1-3]. The PST action for 2-form gauge field in $\mathrm{D}=6$ can be found in eq. (2.11); see (5.8) for the case of $p$-form gauge fields in $M^{2 p+2}$. The PST action for $2 \mathrm{~d}$ chiral bosons [19] is presented in (4.4).

- HT is used for Henneaux-Teitelboim action (2.25) [6]. 
- PS is used for Perry-Schwarz action (2.26) [7].

- FJ is used for Floreanini-Jackiw action [20] which can be found in eq. (4.13).

\section{PST Lagrangian for 6D selfdual gauge fields}

\subsection{2 -form gauge field in $\mathrm{D}=6$}

\subsubsection{Free action for 2-form gauge potential in $M^{1+5}$}

We begin by discussing the theory of free non-chiral 2-form gauge potential

$$
B_{2}=\frac{1}{2} d x^{\nu} \wedge d x^{\mu} B_{\mu \nu}(x)
$$

in six dimensional spacetime. In eq. (2.1) $x^{\mu}$ are local coordinates of $M^{6}, \mu, \nu=0,1, \ldots, 5$, and $\wedge$ denotes the exterior product of differential forms, $d x^{\mu} \wedge d x^{\nu}=-d x^{\nu} \wedge d x^{\mu}$, so that the second rank tensor $B_{\mu \nu}(x)$ contributing to (2.1) is antisymmetric, $B_{\mu \nu}(x)=-B_{\nu \mu}(x)$. The standard field strength of the 2 -form gauge potential is

$$
H_{3}=\frac{1}{3 !} d x^{\rho} \wedge d x^{\nu} \wedge d x^{\mu} H_{\mu \nu \rho}(x):=d B_{2}
$$

where $d=d x^{\mu} \partial_{\mu}$ is the exterior derivative which in our notation acts from the right, e.g.

$$
\begin{aligned}
d B_{2} & =\frac{1}{2} d x^{\mu} \wedge d x^{\nu} \wedge d x^{\rho} \partial_{\rho} B_{\nu \mu}(x) \\
& \equiv \frac{1}{3 !} d x^{\mu} \wedge d x^{\nu} \wedge d x^{\rho}\left(\partial_{\rho} B_{\nu \mu}(x)+\partial_{\mu} B_{\rho \nu}(x)+\partial_{\nu} B_{\mu \rho}(x)\right)
\end{aligned}
$$

so that (2.2) implies $H_{\mu \nu \rho}=3 \partial_{[\mu} B_{\nu \rho]}:=\partial_{\mu} B_{\nu \rho}+\partial_{\nu} B_{\rho \mu}+\partial_{\rho} B_{\mu \nu}$.

The free field action for the 2 -form gauge potential reads

$$
S_{0}=\int_{M^{6}} \mathcal{L}^{0}, \quad \mathcal{L}^{0}=\frac{1}{2} H_{3} \wedge * H_{3},
$$

where the Lagrangian 6-form $\mathcal{L}^{0}$ is written using the Hodge star symbol,

$$
* H_{3}:=\frac{1}{3 !} d x^{\rho} \wedge d x^{\mu} \wedge d x^{\nu}(* H)_{\mu \nu \rho}, \quad(* H)^{\mu \nu \rho}:=\frac{1}{3 ! \sqrt{|g|}} \varepsilon^{\mu \nu \rho \mu^{\prime} \nu^{\prime} \rho^{\prime}} H_{\mu^{\prime} \nu^{\prime} \rho^{\prime}},
$$

$\varepsilon^{\mu \nu \rho \mu^{\prime} \nu^{\prime} \rho^{\prime}}=\varepsilon^{\left[\mu \nu \rho \mu^{\prime} \nu^{\prime} \rho^{\prime}\right]}$ is the Levi-Civita antisymmetric tensor density normalized so that $\varepsilon^{012345}=1$ and $g=\operatorname{det}\left(g_{\mu \nu}\right)$ is the determinant of the spacetime metric which in our conventions is of mostly minus signature.

Varying the action (2.4) with respect to the gauge potential, one finds the standard free 'Maxwell-like' equations for the 3-form field strength

$$
d * H_{3}=0
$$

By construction, the field strength also obeys the Bianchi identities,

$$
d H_{3}=0 \text {. }
$$


In spacetime of nontrivial topology, (2.2) generically gives only a particular solution of (2.7). The general solution

$$
H_{3}=d B_{2}+k_{L} \Omega_{3}^{L}, \quad d \Omega_{3}^{L}=0, \quad \Omega_{3}^{L} \neq d \Xi_{2}^{L}, \quad L=1, \ldots, b_{3},
$$

contains a topological contribution $k_{L} \Omega_{3}^{L}$ determining the cohomological class $\left[H_{3}\right]$ of $H_{3}$ as $\left[H_{3}\right]=k_{L}\left[\Omega^{L}\right]$. Here $\Omega_{3}^{L}$ is the basis of the 3 rd cohomology group $\mathbb{H}^{3}\left(M^{6}\right)$ of the spacetime $M^{6}, b_{3}=\operatorname{dim}^{3}\left(M^{6}\right)$, and $k_{L}$ are constant coefficients (see e.g. [21]).

Despite the presence of topological contributions, the variational problem is usually defined within a fixed cohomology class (see [22, 23] and e.g. [10]), this is to say

$$
\delta H_{3}=d \delta B_{2}
$$

with an arbitrary $\delta B_{2}$.

\subsection{2 (Anti-)self duality equation}

A particular solution of the second order equation (2.6) is provided by 3 -form field strength obeying the anti-self-duality condition

$$
H_{3}+* H_{3}=0 \quad \Leftrightarrow \quad H_{\mu \nu \rho}+\frac{\sqrt{|g|}}{3 !} \varepsilon_{\mu \nu \rho \mu^{\prime} \nu^{\prime} \rho^{\prime}} H^{\mu^{\prime} \nu^{\prime} \rho^{\prime}}=0 .
$$

Clearly, this first order equation is not satisfied by the most general solution of (2.6). Furthermore, the action (2.4) vanishes on this solution. (The above statements are also valid for the self-duality condition $H_{3}-* H_{3}=0$ ).

It was natural to wander whether it is possible to construct the action which produces just the (anti-)self duality equation, or dualities between the field strengths of different ranks, as equations of motion. The action principle of such a type was of great interest for the development of supersymmetry and string theory as far as many important supermultiplets, such as the ones of 10D type IIB supergravity and of the M-theory 5-brane, included self-dual and/or anti-self-dual tensor fields. Such duality invariant actions with broken Lorentz symmetry (better to say, which are not-manifestly Lorentz invariant [19]) were proposed in $[29]$ and in $[6,7]$. The covariant action principle was developed in $[1,2] .^{2}$ The properties of this PST action in spacetime of nontrivial topology is our main interest in this paper.

\footnotetext{
${ }^{2}$ Notice also the existence of a covariant action principle with infinitely many auxiliary fields [24, 25] which describes just the gauge fields obeying (anti-)self duality equations if the additional restrictions to the configurations containing only finite number of fields is imposed. A quite curious nonlocal approach to chiral bosons was proposed in [26]; it implies $1+5$ splitting of $6 \mathrm{~d}$ coordinates and the canonical brackets for $6 \mathrm{~d}$ 2 -form potentials is the same as in HT approach, but its relation with HT action [6] is not clear for us. There exists also a pragmatic approach in the frame of which the (anti-)self-duality or/and duality equations do not follow from the action but are imposed by hand afterwards. In such a way the 'democratic' formulation of 10D type IIA and IIB supergravities [27] and the alternative 5-brane action of [28] were constructed. However, for instance, switching on interactions in a covariant approach of this type does not look so straightforward as when having the action principle of canonical type, like [4, 5, 13, 14, 16].
} 


\subsection{Duality invariant action for the 2-form gauge field. PST, HT and PS actions; $d a$-timelike and $d a$-spacelike branches of PST system}

\subsubsection{PST action}

The standard PST Lagrangian 6-form

$$
\begin{aligned}
\mathcal{L}_{6}^{P S T}=-\left(i_{v} * H_{3}+i_{v} H_{3}\right) \wedge H_{3} \wedge v & = \\
& =\frac{1}{2} d^{6} x \sqrt{|g|} v^{\rho}\left(* H_{\mu \nu \rho}+H_{\mu \nu \rho}\right) * H^{\mu \nu \lambda} v_{\lambda},
\end{aligned}
$$

is defined on any 6-dimensional manifold $M^{6}$ which allows for the existence of nowhere vanishing vector field; it is real when spacetime has a metric of Lorentz signature, $M^{6}=$ $M^{1+5}$.

In (2.11) $x^{\mu}$ are local coordinates on $M^{6},|g|=\left|\operatorname{det}_{\mu \nu}\right|$,

$$
v=d x^{\mu} v_{\mu}=\frac{d a}{\sqrt{|\partial a \partial a|}}, \quad \partial a \partial a:=\partial_{\mu} a g^{\mu \nu}(x) \partial_{\nu} a,
$$

where $a(x)$ is a(n auxiliary) scalar field, called PST scalar, $* H_{3}$ is defined in (2.5), and

$$
i_{v} H_{3}=\frac{1}{2} d x^{\rho} \wedge d x^{\nu} v^{\mu} H_{\mu \nu \rho}(x),
$$

is the contraction of the three form (2.2) with the vector field $v^{\mu}=g^{\mu \nu} v_{\nu}$ dual to the one form $v$ in (2.12). Notice that, by definition,

$$
v^{2}=v^{\mu} v_{\mu}= \pm 1
$$

As we discuss below (and as we have already mentioned in the Introduction) the sign plus corresponds to the da-timelike branch and the sign minus to the da-spacelike branch of the PST system.

In the spacetime of nontrivial topology it may be convenient to consider $d a(x)$ as closed but not exact 1-form, this is to say to consider $a$ as an angle variable (which implies an equivalence relation of the type $a \sim a+2 \pi)$. This is consistent as $a(x)$ enters the action under derivative so that the constant shift of its value, $a(x) \mapsto a(x)+$ const is a symmetry of the action.

When working with the differential form representation, it is convenient to use the identities

$$
\begin{aligned}
& F_{p} \equiv i_{v} F_{p} \wedge v / v^{2}+*\left(i_{v} * F_{p} \wedge v\right) / v^{2}, \quad F_{6}=i_{v} F_{6} \wedge v / v^{2}, \\
& i_{v} * \mathcal{H}_{3} \equiv *\left(\mathcal{H}_{3} \wedge v\right),
\end{aligned}
$$

${ }^{3}$ and $F_{p} \wedge * G_{p}=G_{p} \wedge * F_{p}$. We can also write the first equation in (2.15) as

$$
F_{p} \equiv i_{v} F_{p} \wedge \frac{v}{v^{2}}+F_{p}^{(-)}, \quad i_{v} F^{(-)}=0, \quad F_{p}^{(-)}=\frac{*\left(i_{v} * F_{p} \wedge v\right)}{v^{2}} .
$$

In the case of $d a=d t, F_{p}^{(-)}$is a $p$-form on the spacial slice $M_{t}^{5}$ of the spacetime $M^{1+5}$.

\footnotetext{
${ }^{3}$ To have $* *=1$ we define $* B_{2}=-\frac{1}{4 !} d x_{4}^{\rho} \wedge \ldots \wedge d x_{1}^{\rho} \frac{\sqrt{|g|}}{2} \epsilon_{\rho_{1} \ldots \rho_{4} \mu \nu} B^{\mu \nu}$ while keeping the plus sign in the definition $* G_{4}=\frac{1}{2} d x^{\nu} \wedge d x^{\mu} \frac{\sqrt{|g|}}{4 !} \epsilon_{\mu \nu \rho_{1} \ldots \rho_{4}} G^{\rho_{1} \ldots \rho_{4}}$. This provides the plus sign in (2.16).
} 


\subsubsection{Lagrangian equations from PST action}

We begin by reviewing the properties of the PST action $\propto \int_{M^{6}} \mathcal{L}_{6}^{P S T}$ in the case of topologically trivial spacetime.

The variation of $\mathcal{L}_{6}^{P S T}$ reads

$$
\delta \mathcal{L}_{6}^{P S T}= \pm 2 \mathcal{G}_{2} \wedge d a \wedge\left(\delta H_{3}-\frac{1}{2} d(\delta a) \wedge \mathcal{G}_{2}\right) \mp H_{3} \wedge \delta H_{3}
$$

where the sign $\mp$ in the last term corresponds to $\pm 1=v^{2}$ and

$$
\mathcal{G}_{2}:=\frac{i_{v}\left(* H_{3}+H_{3}\right)}{\sqrt{\partial a \partial a}} .
$$

As we are interested in $H_{3}=d B_{2}$, or (2.8) but with the variation within a fixed topological class (2.9),

$$
\delta \mathcal{L}_{6}^{P S T}=2 \mathcal{G}_{2} \wedge d a \wedge\left(d \delta B_{2}-\frac{1}{2} d(\delta a) \wedge \mathcal{G}_{2}\right) \mp d\left(H_{3} \wedge \delta B_{2}\right)
$$

For the case of spacetime without boundary, $\partial M^{6}=0$, the last term does not contribute into the variation of the action $S^{P S T} \propto \int_{M^{6}} \mathcal{L}_{6}^{P S T}$.

Now it is easy to see that the Lagrangian equation of motion for the 2-form potential, which follows from the PST action, reads

$$
d\left(\mathcal{G}_{2} \wedge d a\right)=0 .
$$

The equation of motion for the PST scalar have the form

$$
\mathcal{G}_{2} \wedge d\left(\mathcal{G}_{2} \wedge d a\right)=0
$$

Clearly this is satisfied identically due to (2.21). This dependence of the $\delta a$ equation makes transparent the pure gauge (Stückelberg) nature of the PST scalar; in other words, it is the Noether identity which manifests the presence of the gauge symmetry (called PST2 gauge symmetry) with respect to arbitrary variations $\delta a(x)$ of the PST scalar supplemented by a suitable variation of $B_{2}$ (the second term in (2.24) below).

But this is not the end of story. Eqs. (2.21) can be solved formally with respect to $\mathcal{G}_{2}$. In the case of topologically trivial spacetime $M^{6}$ (with $b_{2}=0$ and $b_{3}=0$ ) the solution is

$$
\mathcal{G}_{2} \wedge d a=-d\left(\phi_{1} \wedge d a\right)
$$

where $\phi_{1}=d x^{\mu} \phi_{\mu}(x)$ is an arbitrary 1-form.

\subsubsection{Gauge symmetries and branches of the PST system}

Now we come back to eq. (2.20) and observe that it also makes manifest the gauge symmetries which act on the 2 -form potential by

$$
\delta B_{2}=\varphi_{1} \wedge d a+\delta a \mathcal{G}_{2} .
$$


Here $\varphi_{1}=d x^{\mu} \varphi_{\mu}(x)$ and $\varphi_{\mu}(x)$ is an arbitrary $\mathrm{x}$-dependent vector function parametrizing the PST1 gauge symmetry and $\delta a=\delta a(x)$ is an arbitrary $\mathrm{x}$-dependent variation of the PST scalar $a(x)$ (parametrizing the PST2 gauge symmetry).

Roughly speaking, the PST2 gauge symmetry can be used to gauge $a(x)$ away. However, not all the gauges are admissible as far as the presence of $\sqrt{\partial a \partial a}$ in the denominator of the Lagrangian, and of the equations of motion as they obtained from the variation of the action (Lagrangian equations), put some topological restrictions on the PST scalar. Neither $a(x)=$ const (which implies $\partial_{\mu} a=0$ ) nor identification of $a(x)$ with the combination of coordinate parametrizing a light-like direction (for which $\sqrt{|\partial a \partial a|}=0$ ) is allowed. However, the configurations where $a(x)$ is identified with coordinate in timelike or a spacelike direction are not forbidden.

In the case when $\partial_{\mu} a$ is a timelike vector, $\partial a \partial a:=\partial_{\mu} a g^{\mu \nu} \partial_{\nu} a>0$, the PST2 gauge symmetry of the PST action can be used to fix the gauge $a(x)=x^{0}=t$ (or better $d a(x)=d t)$. In the case of the PST action with spacelike $\partial_{\mu} a, \partial a \partial a<0$, we can use the PST2 gauge symmetry to fix the gauge $a(x)=x^{5}$ (better $\left.d a(x)=d x^{5}\right)$. We will call these da-timelike and da-spacelike branches of the PST system, respectively. These branches are disconnected as far as the configurations $a(x)=t$ and, say, $a(x)=x^{5}$ cannot be related by a smooth PST2 transformation. ${ }^{4}$

\subsubsection{Gauge fixed form of the PST action: HT and PS actions}

Fixing the gauge $d a=d t$ in da-timelike branch of the PST system we arrive at non-manifestly Lorentz- invariant (non-manifestly diffeomorphism invariant) HenneauxTeitelboim action $[6,31]$

$$
S^{H T} \propto \int_{M^{6}} \mathcal{L}_{6}^{H T}, \quad \mathcal{L}_{6}^{H T}=-\mathcal{G}_{2}^{H T} \wedge H_{3} \wedge d t, \quad \mathcal{G}_{2}^{H T}=i_{0}\left(H_{3}+* H_{3}\right) .
$$

On the other hand, fixing the gauge (say) $d a=d x^{5}$ in $d a$-spacelike branch results in the Perry-Schwarz action [7]

$$
S^{P S} \propto \int_{M^{6}} \mathcal{L}_{6}^{P S}, \quad \mathcal{L}_{6}^{P S}=-\mathcal{G}_{2}^{P S} \wedge H_{3} \wedge d x^{5}, \quad \mathcal{G}_{2}^{P S}=i_{5}\left(H_{3}+* H_{3}\right) .
$$

It is easy to write the (counterparts of the) PST1 gauge symmetry leaving invariant (2.25) and (2.26):

$$
\begin{array}{lll}
\delta B_{2}=\varphi_{1} \wedge d t & \Rightarrow & \delta S^{H T}=0, \\
\delta B_{2}=\varphi_{1} \wedge d x^{5} & \Rightarrow & \delta S^{P S}=0 .
\end{array}
$$

\footnotetext{
${ }^{4}$ Indeed, let us define one-parametric family of the configurations $a(x)=(1-\alpha) t+\alpha x^{1}$, which coincides with $a(x)=t$ and $a(x)=x^{1}$ for $\alpha=0$ and $\alpha=1$, respectively. The fact that $\alpha=1 / 2$ representative of this family corresponds the prohibited configuration $a(x)=1 / 2\left(t+x^{1}\right)$, for which $\partial a \partial a=0$ and the action and the Lagrangian equations become singular, indicates that the PST2 transformation relating the $a(x)=t$ and $a(x)=x^{1}$ configurations is inevitably singular. This shows that, even in a topologically trivial situation, the dynamical system described by the PST action has two disconnected branches, which we call da-timelike and da-spacelike branches of the PST system.
} 


\subsubsection{Anti-self-duality from Lagrangian equations of motion}

Under the PST1 gauge symmetry $\delta H_{3}=d\left(\varphi_{1} \wedge d a\right)$ and $\delta \mathcal{G}_{2} \wedge d a=-d\left(\varphi_{1}\right) \wedge d a$ so that, making this transformation in (2.23) and choosing $\varphi_{1}=\phi_{1}$ we arrive at $\mathcal{G}_{2}=0$ which is equivalent to the anti-self-duality condition (2.10),

$$
\mathcal{G}_{2}=0 \quad \Leftrightarrow \quad H_{3}+* H_{3}=0
$$

In the same manner one can proceed in the case of HT and PS actions (2.25) and (2.26).

Notice that there exists another, although equivalent, way of reasonings, which is often used in discussion of not manifestly invariant HT and PS actions. First one observes that the symmetry (2.27) of the HT action (2.25) actually implies that $B_{0 i}$ component is absent in it. In other words, $\mathcal{L}_{6}^{H T}$ contains $B_{i j}=-B_{j i}$ fields only,

$$
\mathcal{L}_{6}^{H T}=-i_{0}\left(d B_{2}^{(-)}+*\left(d B_{2}^{(-)}\right)\right) \wedge d B_{2}^{(-)} \wedge d t, \quad B_{2}^{(-)}=\frac{1}{2} d x^{i} \wedge d x^{j} B_{j i}(x) .
$$

Varying with respect to these fields we find

$$
d\left(\mathcal{G}_{2}^{(-)} \wedge d t\right)=0, \quad \mathcal{G}_{2}^{(-)}=i_{0}\left(d B_{2}^{(-)}+* d B_{2}^{(-)}\right) .
$$

Notice that $\left(d B_{2}^{-}\right)_{i j k}=H_{i j k}:=\left(d B_{2}\right)_{i j k}$ does not feel presence or absence of $B_{0 j}$ component so that $i_{0}\left(* d B_{2}^{-}\right)=i_{0}\left(* d B_{2}\right)$ and we can write the Lagrangian equations of motion (2.31) in the following first order form (cf. (2.23))

$$
\mathcal{G}_{2}^{(-)} \wedge d t=d \phi_{1} \wedge d t \quad \Leftrightarrow \quad\left(d B_{2}^{(-)}\right)_{0 i j}+\left(* d B_{2}\right)_{0 i j}=\partial_{i} \phi_{j}-\partial_{j} \phi_{i}
$$

This includes $\left(d B_{2}^{-}\right)_{0 i j}=\partial_{0} B_{i j}$ while the complete $\left(d B_{2}\right)_{0 i j}=\partial_{0} B_{i j}-2 \partial_{[i \mid} B_{0 \mid j]}$ would involve $B_{0 j}$ absent in (2.30). The next, final observation is that we can identify the component $B_{0 i}$, absent in the HT action (2.25), with the arbitrary $\phi_{i}$ appeared in the solution (2.32),

$$
B_{0 i}=\phi_{i},
$$

after which (2.32) acquires the form $\left(d B_{2}\right)_{0 i j}+\left(* d B_{2}\right)_{0 i j}=0$ and implies the anti-self duality equation for the standard field strength

$$
\left.\left(d B_{2}\right)_{0 i j}\right|_{B_{0 i}=\phi_{i}}+\frac{1}{3 !} \epsilon_{i j k l m}\left(d B_{2}\right)^{k l m}=0, \quad \Rightarrow H_{3}+* H_{3}=0, \quad H_{3}=\left.d B_{2}\right|_{B_{0 i}=\phi_{i}} .
$$

It is useful to keep in mind this alternative way of thinking on the derivation of anti-selfduality relation from the HT action, and similar approach to the PS formulation. However, let us stress that the two forms of HT action, (2.25) and (2.30), and two forms of equations of motion, (2.32) and (2.10) ((2.34)), are equivalent because the arbitrary variation of $B_{0 i}$ 'parametrizes' a gauge symmetry of (2.25).

Summarizing, as we have reviewed above, in topologically trivial spacetime $M^{6}=$ $M^{1+5}$, the anti-self-duality equation (2.10) can be obtained as a gauge fixed version of the Lagrangian equation of motion (2.21) which follows from the PST action (2.11). The main question we address here is whether this is also the case when the spacetime topology is nontrivial. 


\section{PST action and equations of motion in topologically nontrivial $M^{1+5}$}

A topologically nontrivial spacetime manifold $M^{6}$ may have some number $b_{2}$ of closed but not exact 2 forms $\omega_{2}^{\Lambda}$,

$$
d \omega_{2}^{\Lambda}=0, \quad \omega_{2}^{\Lambda} \neq d \chi_{1}^{\Lambda}, \quad \Lambda=1, \ldots, b_{2},
$$

and also some number $b_{3}$ of closed but not exact 3 -forms $\Omega_{3}^{L}$

$$
d \Omega_{3}^{L}=0, \quad \Omega_{3}^{L} \neq d \Xi_{2}^{L}, \quad L=1, \ldots, b_{3}
$$

(see (2.8)). The number of these closed forms, $b_{2}$ and $b_{3}$, determining the dimensions of the second and the third de Rahm cohomology groups of the spacetime manifold $M^{6}$, are called the second and third Betti numbers of $M^{6}$,

$$
b_{2}=\operatorname{dim} \mathbb{H}^{2}\left(M^{6}\right), \quad b_{3}=\operatorname{dim} \mathbb{H}^{3}\left(M^{6}\right) .
$$

The PST action is well defined if $M^{6}$ admits a nowhere vanishing vector field. As the existence of at least one such field is necessary condition for a manifold to admit Lorentz-type metric, the PST action always makes sense in $M^{1+5}$ with nonsingular metric of Lorentzian signature. However, if on $M^{1+5}$ under consideration such a nowhere vanishing vector field is unique, then both $d t$ and the derivative of PST scalar $d a(x)$ should be identified with a dual of that; as a result, $d a(x)=d t$ and the PST action coincides with the HT one on such a manifold. The difference between PST and HT action occurs when several (more than one) nowhere vanishing vector fields can exist on $M^{6}=M^{1+5}$. Simplest examples of such spaces are given by direct products of flat spacetime with an arbitrary internal manifold, $\mathbb{R}^{1+n} \otimes M^{5-n}$ with $5 \geq n \geq 1$.

\subsection{First order form of the PST Lagrangian equations in a topologically non- trivial spacetime}

In a topologically nontrivial $M^{6}$, as far as the Bianchi identities (2.7) are still valid and we use the variation within fixed topological class $\delta H_{3}=d \delta B_{2}$, eq. (2.9), the variation of the Lagrangian form is given by (2.20), and the Lagrangian equations of motion keep the same form (2.21),

$$
d\left(\mathcal{G}_{2} \wedge d a\right)=0
$$

However, when resolving them with respect to $\mathcal{G}_{2}$ (defined in (2.19)), we first find

$$
\mathcal{G}_{2} \wedge d a=d \phi_{2}+\tilde{k}_{L} \Omega_{3}^{L} .
$$

with constant $\tilde{k}_{L}$ and arbitrary 2 -form $\phi_{2}$. To proceed, we need to project this differential form equation into the part which contains $d a$ and its complementary, which does not contain $d a$. Although this can be done with generic $d a$, the discussion becomes much more transparent if we consider the case $d a=d t$, in which eq. (3.5) acquires the form

$$
\mathcal{G}_{2} \wedge d t=d \phi_{2}+\tilde{k}_{L} \Omega_{3}^{L}, \quad \mathcal{G}_{2}=i_{0}\left(H_{3}+* H_{3}\right) .
$$


Let us decompose the forms and differential on the pure spacial and $d t$ dependent parts,

$$
\begin{aligned}
d & =d^{(-)}+d t \partial_{t}, \quad d^{(-)}=d \vec{x} \vec{\partial}, \\
\phi_{2} & =\phi_{2}^{(-)}+i_{0} \phi_{2} \wedge d t=\phi_{2}^{(-)}+\phi_{1} \wedge d t, \\
\omega_{2}^{\Lambda} & =\omega_{2}^{\Lambda(-)}+i_{0} \omega_{2}^{\Lambda} \wedge d t \\
\Omega_{3}^{L} & =\Omega_{3}^{L(-)}+i_{0} \Omega_{3}^{L} \wedge d t .
\end{aligned}
$$

With splitting (3.9) and (3.10), the closure of basic 2-forms and 3-forms, eqs. (3.1) and (3.2), imply that the pure spacial part $\left({ }^{(-)}\right.$part) of the closed forms should be 'spatially closed', or $d^{(-)}$-closed forms, and $\partial_{t}$ derivatives of the spacial $\left(^{(-)}\right)$part of the closed forms should be $d^{(-)}$-exact,

$$
\begin{array}{ll}
d^{(-)} \omega_{2}^{\Lambda(-)}=0, & \partial_{t} \omega_{2}^{\Lambda(-)}=d^{(-)} i_{0} \omega_{2}^{\Lambda}, \\
d^{(-)} \Omega_{3}^{L(-)}=0, & \partial_{t} \Omega_{3}^{L(-)}=d^{(-)} i_{0} \Omega_{3}^{L} .
\end{array}
$$

Decomposing in the same manner eq. (3.6) we find

$$
\begin{aligned}
\mathcal{G}_{2} \wedge d t & =d^{(-)} \phi_{1} \wedge d t+\left(\partial_{t} \phi_{2}^{(-)}+\tilde{k}_{L} i_{0} \Omega_{3}^{L}\right) \wedge d t \\
d^{(-)} \phi_{2}^{(-)}+\tilde{k}_{L} \Omega_{3}^{L(-)} & =0 .
\end{aligned}
$$

Taking into account eqs. (3.12), we see that (3.14) implies that 2 -form $\partial_{t} \phi_{2}^{(-)}+\tilde{k}_{L} i_{0} \Omega_{3}^{L}$ is $d^{(-)}$closed, $d^{(-)}\left(\partial_{t} \phi_{2}^{(-)}+\tilde{k}_{L} i_{0} \Omega_{3}^{L}\right)=0$ so that it can be decomposed into a sum of a $d^{(-)}$-exact form and a $d^{(-)}$-closed but not $d^{(-)}$exact 2 -form:

$$
\left(\partial_{t} \phi_{2}^{(-)}+\tilde{k}_{L} i_{0} \Omega_{3}^{L}\right)=d^{(-)} \check{\phi}_{1}^{(-)}+\check{\omega}_{2}^{(-)}(t, \vec{x}), \quad\left\{\begin{array}{l}
d^{(-)} \check{\omega}_{2}^{(-)}(t, \vec{x})=0, \\
\check{\omega}_{2}^{(-)}(t, \vec{x}) \neq d^{(-)} \chi_{1}^{(-)}(t, \vec{x}) .
\end{array}\right.
$$

When substituting this into (3.13), the exact form can be absorbed into the first term, in which $d^{(-)}$can be equivalently substituted by $d$, so that the result reads

$$
\mathcal{G}_{2} \wedge d t=-d \phi_{1} \wedge d t+\check{\omega}_{2}^{(-)}(t, \vec{x}) \wedge d t, \quad\left\{\begin{array}{l}
d^{(-)} \check{\omega}_{2}^{(-)}(t, \vec{x})=0, \\
\check{\omega}_{2}^{(-)}(t, \vec{x}) \neq d^{(-)} \chi_{1}^{(-)}(t, \vec{x}) .
\end{array}\right.
$$

A nontrivail topology of $M^{6}$ may manifest itself in the second term in right hand side (r.h.s.) of this equation.

As one can see from (3.11), a particular case of $d^{(-)}$closed but not $d^{(-)}$exact $\check{\omega}_{2}^{(-)}(t, \vec{x})$ is provided by pure spacial components of closed but not exact forms $\omega_{2}^{\Lambda}$, possibly multiplied by an arbitrary function of $t$. In general

$$
\check{\omega}_{2}^{(-)}(t, \vec{x})=l_{\Lambda}(t) \omega_{2}^{\Lambda(-)}+\tilde{\omega}_{2}^{(-)}(t, \vec{x}), \quad d^{(-)} \tilde{\omega}_{2}^{(-)}(t, \vec{x})=0, \quad \partial_{t} \tilde{\omega}_{2}^{(-)}(t, \vec{x}) \neq d^{(-)} \chi_{1}^{(-)}
$$

with $b_{2}$ arbitrary functions $l_{\Lambda}(t)$ of time coordinate only and spatially closed $\tilde{\omega}_{2}^{(-)}(t, \vec{x})$ the time derivative of which is not $d^{(-)}$-exact (so that, in distinction with $\omega_{2}^{\Lambda(-)}$, this is not a spacial part of a closed form in $M^{1+5}$ ). 
In the case of $M^{1+6}=\mathbb{R}^{1} \otimes M^{5}$ all the nontrivial forms have only spacial parts, in particular $\omega_{2}^{\Lambda}=\omega_{2}^{\Lambda(-)}(\vec{x})$, so that all the $\check{\omega}_{2}^{(-)}=\check{\omega}_{2}^{(-)}(\vec{x})$ is decomposed on these, the last term is absent, $\tilde{\omega}_{2}^{(-)}=0$, and eq. (3.16) reads

$$
\mathcal{G}_{2} \wedge d t=d \phi_{1} \wedge d t+l_{\Lambda}(t) \omega_{2}^{\Lambda} \wedge d t
$$

In this suggestive case, which we will widely use in our discussion below, it becomes especially transparent that the presence or absence of nontrivial 3 -forms $\Omega_{3}^{L}$ ( $b_{3} \neq 0$ versus $b_{3}=0$ ) is not important when studying the consequence of the Lagrangian PST equation. What does matter is the possible presence of closed but not exact 2 forms in the spacetime $\left(b_{2} \neq 0\right.$ versus $\left.b_{2}=0\right)$ and on its 5 dimensional slices (spacial slices $M_{t}^{5}$ in the case under consideration).

When $d a$ is not identified with $d t$, one can also arrive at the counterpart of eq. (3.16)

$$
\mathcal{G}_{2} \wedge d a=-d \phi_{1} \wedge d a+\check{\omega}_{2} \wedge d a, \quad d \check{\omega}_{2}=\check{\omega}_{2}^{(1)} \wedge d a
$$

Here $\check{\omega}_{2}^{(1)}$ can be considered as an arbitrary 2-form which can be identified with $\frac{d}{d a} \check{\omega}_{2}$. However, the consistency conditions of the second equation in (3.19) require it to obey the equation of the same structure $d \check{\omega}_{2}^{(1)}=\check{\omega}_{2}^{(2)} \wedge d a$. This chain is continued up to infinity $\left(d \check{\omega}_{2}^{(2)}=\check{\omega}_{2}^{(3)} \wedge d a, \ldots, d \check{\omega}_{2}^{(n)}=\check{\omega}_{2}^{(n+1)} \wedge d a, \ldots\right)$ and represents a counterpart of $d^{(-)} \breve{\omega}_{2}=0$ condition in (3.16) (actually it can be equivalently written in this form provided $d^{(-)} \check{\omega}_{2}=d \check{\omega}_{2} \mp i_{v} d \check{\omega}_{2} \wedge v$ with $\left.v \propto d a(2.12)\right)$.

In some cases (for some $M^{6}$ ) we can write eq. (3.19) in the form similar to (3.18),

$$
\mathcal{G}_{2} \wedge d a=d \phi_{1} \wedge d a+l_{\Lambda}(a(x)) \omega_{2}^{\Lambda} \wedge d a(x), \quad \Lambda=1, \ldots, b_{2},
$$

In addition to the arbitrary 1 -form $\phi_{1}$, the r.h.s. of this equation contains a topological contributions determined by $b_{2}$ arbitrary functions of the PST scalar, $l_{\Lambda}(a)=l_{\Lambda}(a(x))$. As in the case of topologically trivial $M^{6}$, the first term can be gauged away using the PST1 gauge symmetry, but the fate of the second term, containing topological contribution, have to be studied.

\section{2 'Semilocal symmetry' of the PST action in topologically nontrivial $M^{6}$}

Coming back to the variation of the Lagrangian form, eq. (2.20), we find that in a topologically nontrivial spacetime $M^{6}=M^{1+5}$ it vanishes under the transformations of the PST2 gauge symmetry $\delta B_{2}=\delta a(x) \mathcal{G}_{2}$ (which are the same as in the topologically trivial case) and also under the variations $\delta H_{3}=d \delta B_{2}$ which obey

$$
d a \wedge d \delta B_{2} \equiv d\left(\delta B_{2} \wedge d a\right)=0
$$

Actually this equation for $\delta B_{2}^{(-)}:=\delta B_{2} \mp i_{v} \delta B_{2} \wedge v$ (with $v \propto d a$, eq. (2.12)) have the same structure as eq. (3.4) for $\mathcal{G}_{2} \propto i_{v}\left(H_{3}+* H_{3}\right)$. Hence, using the results of previous subsection, we can immediately write its general solution for $\delta B_{2}$. Combining it with the above mentioned PST2 transformations (and ignoring the conventional gauge symmetry $\left.\delta B_{2}=d \alpha_{1}\right)$, we find

$$
\delta B_{2}=\varphi_{1} \wedge d a+\delta a \mathcal{G}_{2}+\check{\varphi}_{2}, \quad d \check{\varphi}_{2}=\check{\varphi}_{2}^{(1)} \wedge d a
$$


which, at least in some particular cases (see above) can be written as

$$
\delta B_{2}=\varphi_{1} \wedge d a+\delta a \mathcal{G}_{2}+\omega_{2}^{\Lambda} f_{\Lambda}(a(x))
$$

(see (3.1) for properties of $\omega_{2}^{\Lambda}$ ).

In addition to an arbitrary $\mathrm{x}$-dependent one form variation $\varphi_{1}=d x^{\mu} \varphi_{\mu}(x)$, and an arbitrary variation of the PST scalar $\delta a(x)$ (parametrizing the PST1 and PST2 gauge symmetries, respectively), this contains $b_{2}$ arbitrary functions of the PST scalar field $a=$ $a(x), f_{\Lambda}(a)=f_{\Lambda}(a(x))$. These parametrize transformations of semilocal symmetry.

In general case the semi-local symmetry is described by two-form $\check{\varphi}_{2}$ in (3.22) which obeys $d \check{\varphi}_{2}=\check{\varphi}_{2}^{(1)} \wedge d a$; as such a description seems to be less transparent, it is very useful to keep in mind the particular case described above.

To gain more comprehension of the properties of our system, let us discuss the configurations with $d a=d t$ of the $d a$-timelike branch of the PST system, or the case of HT action. Then (3.22) acquires the form

$$
\delta B_{2}=\varphi_{1} \wedge d t+\check{\varphi}_{2}^{(-)}, \quad d^{(-)} \check{\varphi}_{2}^{(-)}=0,
$$

and (3.23), valid in particular cases (including $\left.M^{6}=\mathbb{R}^{1} \otimes M^{5}, 5\right)$, reads

$$
\delta B_{2}=\varphi_{1} \wedge d t+\omega_{2}^{\Lambda} f_{\Lambda}(t) .
$$

We see that in these cases the semilocal symmetry transformations are parametrized by $b_{2}$ functions of time variables only, $f_{\Lambda}(t)$. In general case of topologically nontrivial spacetime $M^{1+5}$, the parameters of the semi-local symmetry of HT action are hidden inside a $t$ dependent $d^{(-)}$-closed but not $d^{(-)}$-exact 2 -form, $\check{\varphi}_{2}^{(-)}(t, \vec{x})$ in $(3.24)$.

The symmetry transformations of the field strength $H_{3}=d B_{2}$ read

$$
\delta H_{3}=d\left(\varphi_{1} \wedge d t\right)-\partial_{t} \check{\varphi}_{2}^{(-)} \wedge d t, \quad d^{(-)} \check{\varphi}_{2}^{(-)}(t, \vec{x})=0,
$$

and, in the particular case,

$$
\delta H_{3}=d\left(\varphi_{1} \wedge d t\right)+\dot{f}_{\Lambda}(t) \omega_{2}^{\Lambda} \wedge d t
$$

where $\dot{f}_{\Lambda}(t):=\frac{d f_{\Lambda}(t)}{d t}$. As it is easily to see, $i_{0} * \delta H_{3}=i_{0} \delta * H_{3}=0$, so that

$$
\delta \mathcal{G}_{2} \wedge d t=-d\left(\varphi_{1}\right) \wedge d t+\partial_{t} \check{\varphi}_{2}^{(-)} \wedge d t, \quad d^{(-)} \check{\varphi}_{2}^{(-)}(t, \vec{x})=0
$$

Eq. (3.28) implies that, as in the topologically trivial case, we can gauge away $\phi_{1}$ in (3.16) using the second PST symmetry with the one-form parameter $\varphi_{1}$. Furthermore, the second term in (3.16) can be also removed by the 'semi-local symmetry' if we choose $\check{\varphi}_{2}^{(-)}(t, \vec{x})$ to be a solution of $\partial_{t} \check{\varphi}_{2}^{(-)}=-\check{\omega}_{2}^{(-)}(t, \vec{x})$.

In particular cases when $\check{\varphi}_{2}^{(-)}(t, \vec{x})$ and $\check{\omega}_{2}^{(-)}(t, \vec{x})$ can be expressed as a linear combination of $\omega_{2}^{(-) \Lambda}(t, \vec{x})$, the above arguments can be formulated in a more transparent manner:

\footnotetext{
${ }^{5}$ The semi-local symmetry of the HT action in such spacetime was observed in [18].
} 
the r.h.s. (3.18) can be removed by a semi-local symmetry provided the $b_{2}$ functions in (3.25) (and $(3.27)$ ) are chosen such that $\dot{f}_{\Lambda}(t)=-l_{\Lambda}(t)$ holds.

Similar statement is true in the generic case of (both branches of) the PST action with an arbitrary (nowhere vanishing) $d a$. Let us begin from the PST system in particular type of spacetime where the general solution of the Lagrangian PST equation (3.4) can be written in the form of (3.20). The r.h.s. of this equation can be removed by the standard PST gauge symmetries and the semi-local symmetry $\delta B_{2}=\omega_{2}^{\Lambda} f_{\Lambda}(a, \delta)$, eq. (3.23), with $a$-dependent variation $f_{\Lambda}(a)$ obeying $f_{\Lambda}^{\prime}(a):=\frac{d}{d a} f_{\Lambda}(a)=-l_{\Lambda}(a)$.

In general case the r.h.s. of the first order form of the PST Lagrangian equation, eq. (3.19), contains 2 -form $\check{\omega}_{2}$ obeying $d \check{\omega}_{2}=\check{\omega}_{2}^{(1)} \wedge d a$, the r.h.s. of (3.19) can be also removed by the standard PST gauge symmetries and the semi-local symmetry, $\delta B_{2}=\check{\varphi}_{2}$ with $d \check{\varphi}_{2}=\check{\varphi}_{2}^{(1)} \wedge d a$ in $(3.22)$, provided we choose $\check{\varphi}_{2}^{(1)}=-\check{\omega}_{2}$.

Then, if the semilocal symmetry is a gauge symmetry, the above statements imply that eq. (3.19) (eq. (3.20)) is gauge equivalent to $\mathcal{G}_{2}=0$ which, in its turn, is equivalent to the usual anti-self-duality equation $H_{3}+* H_{3}=0$, eq. (2.29).

As we will show in section 3.3 this is the case in the da-timelike branch of the PST system, while in the da-spacelike branch the above semilocal symmetry is an infinitely dimensional global symmetry, similar to $d=2$ conformal symmetry.

To make the above statement intuitively clear, we just mention that the gauge nature of semilocal symmetry in the $d a$-timelike PST system is suggested by observation that in it, after gauge fixing the PST2 symmetry by $d a=d t$, the parameters of the semilocal symmetry (3.22) (or (3.23)) can be collected in functions of time variables; in a particular case these are $b_{2}$ functions $f_{\Lambda}(t)$ (see (3.25)). Indeed, the t-dependence is characteristic for parametric functions of gauge symmetries of one-dimensional systems, and, in a $d \geq 2$ field theory, the dependence on spacial coordinates can be considered as a kind of index, although continuous. In contrast, in the da-spacelike branch the parameters of semilocal symmetry can be collected (after gauge fixing the PST2 symmetry by, say, $d a=d x^{5}$ ) in functions of one of the spacial coordinates, which suggests an infinite dimensional global symmetry nature of semilocal symmetry in this case.

\subsection{On Noether current for semilocal symmetry}

A formal way to distinguish a gauge symmetry from an infinite dimensional rigid symmetry, a typical example of which is provided by the $2 \mathrm{~d}$ conformal symmetry, is to calculate the Noether current $J^{\mu}$ and Noether charge $Q=\int d^{D-1} x J^{0}$. For the gauge symmetry this latter is identically equal to zero, $Q=\int d^{D-1} x J^{0}=0$, while for the rigid symmetry this is not the case (see [30] and refs. therein).

In this section we address the question of whether the semilocal symmetry of the PST action in a topologically nontrivial spacetime, which in some particular cases of $M^{6}$ with $b_{2} \neq 0$ have the form of $\delta B_{2}=\omega_{2}^{\Lambda} f_{\Lambda}(a, \delta)$ in (3.23), is the gauge symmetry.

To streamline the discussion, we can consider the non-manifestly Lorentz invariant Henneaux-Teitelboim (HT) and Perry-Schwarz (PS) actions, eqs. (2.25) and (2.26), which can be obtained by gauge fixing from the PST action in its da-timelike and da-specelike branches, respectively. 
Furthermore, we will begin by discussing these actions in particular classes of topologically nontrivial spacetimes, including $\mathbb{R}^{1} \otimes M^{5}$ and $M^{1+4} \otimes \mathbb{R}^{1}$, for which the semi-local symmetry of these actions can be expressed in terms of $b_{2}$ functions of only time coordinate and of only one spacial coordinate respectively, so that

$$
\begin{aligned}
& \delta S^{H T}=0 \Leftarrow \delta B_{2}=d \alpha_{1}+\varphi_{1} \wedge d t+f_{\Lambda}(t) \omega_{2}^{\Lambda} . \\
& \delta S^{P S}=0 \Leftarrow \delta B_{2}=d \alpha_{1}+\varphi_{1} \wedge d x^{5}+f_{\Lambda}\left(x^{5}\right) \omega_{2}^{\Lambda} .
\end{aligned}
$$

Let us recall that $\omega^{\Lambda}, \Lambda=1, \ldots, b_{2}$ are closed but not exact 2 -forms (3.1) which provide a basis of $\mathbb{H}^{2}\left(M^{6}\right)$ which, in these particular cases, is equal to $\mathbb{H}^{2}\left(M^{5}\right)$ and $\mathbb{H}^{2}\left(M^{1+4}\right)$, respectively.

In the case of HT action for chiral bosons in $\mathbb{R}^{1} \otimes M^{5}$, we can write the semi-local symmetry transformations of (3.29) in the form

$$
\delta B_{2}=\left(f_{\Lambda}(0)+t f_{\Lambda}^{\prime}(0)+\ldots+t^{n} \frac{f_{\Lambda}^{(n)}(0)}{n !}+\ldots\right) \omega_{2}^{\Lambda}
$$

and identify (the infinite set of) their parameters with $\frac{f_{\Lambda}^{(n)}(0)}{n !}$ for $n=0,1, \ldots$. Then the 5 -forms dual to the Noether currents for these symmetries,

$$
* J_{1}^{(n) \Lambda}=\frac{1}{5 !} d x^{\mu_{5}} \wedge \ldots \wedge d x^{\mu_{1}} \epsilon_{\mu_{1} \ldots \mu_{5} \mu} J^{\mu(n) \Lambda} .
$$

read (see appendix B for the proof of the First Noether Theorem in our notation)

$$
* J_{1}^{(n) \Lambda}=t^{n} d t \wedge \mathcal{G}_{2} \wedge \omega_{2}^{\Lambda} .
$$

This form is closed on the mass shell, $d * J_{1}^{(n) \Lambda}=0$, which is tantamount to state the current conservation $\partial_{\mu} J^{\mu(n) \Lambda}=0$. Moreover, one can observe that the time component of the Noether current dual to the 5 -form (3.33) vanishes, $J^{0(n) \Lambda}=0\left(J^{\mu(n) \Lambda}=\delta_{i}^{\mu} J^{i(n) \Lambda}\right)$. Then, as far as the Noether charge is defined as an integral over the space of the timelike component of the Noether current, it also vanishes,

$$
Q^{(n) \Lambda}:=\int d^{5} x J^{0(n) \Lambda}=0,
$$

as it should be for the case of gauge symmetry.

This allows us to conclude that the semi-local symmetry (3.29) is the gauge symmetry of the HT action (2.25) in a spacetime $M^{6}$ of nontrivial topology with $b_{2} \neq 0$.

Such a conclusion does not follow in the case of semi-local symmetry (3.30) of the Perry-Schwarz action $(2.26)$ on $M^{1+4} \otimes \mathbb{R}^{1}$,

$$
\delta B_{2}=\left(f_{\Lambda}(0)+\left(x^{5}\right) f_{\Lambda}^{\prime}(0)+\ldots+\left(x^{5}\right)^{n} \frac{f_{\Lambda}^{(n)}(0)}{n !}+\ldots\right) \omega_{2}^{\Lambda} .
$$

Its Noether current reads

$$
* J_{1}^{(n) \Lambda}=\left(x^{5}\right)^{n} d x^{5} \wedge \mathcal{G}_{2} \wedge \omega_{2}^{\Lambda},
$$


so that in this case $J^{5(n) \Lambda}=0$, but $J^{0(n) \Lambda}$ is generically nonvanishing. Thus the standard Noether charge is generically nonzero, $Q^{(n) \Lambda}:=\int d^{5} x J^{0(n) \Lambda} \neq 0$, which indicates that the semi-local symmetry (3.30) of (2.26) is infinite dimensional rigid symmetry.

The above statement is true also in the case of HT, PS and PST actions in generic $M^{6}$ (allowing for the existence of nowhere vanishing vector field(s)), when the semi-local symmetry is described by eq. (3.22) rather than (3.23). To make this transparent let us first return to the above particular case and introduce generated functionals for the Noether currents and Noether charges,

$$
\begin{aligned}
* J_{1}^{\Lambda}[f(t)] & =\sum_{n=0}^{\infty} \frac{f^{(n)}(0)}{n !} * J_{1}^{(n) \Lambda}=f(t) d t \wedge \mathcal{G}_{2} \wedge \omega_{2}^{\Lambda}, \\
Q^{\Lambda}[f(t)] & =\int d^{5} x J^{0 \Lambda}[f(t)] \equiv \int_{M^{5}} * J_{1}^{\Lambda}[f(t)]=0, \\
Q\left[f_{\Lambda}(t) \omega_{2}^{\Lambda}\right] & =\sum_{\Lambda} Q^{\Lambda}\left[f_{\Lambda}(t)\right]=0, \\
* J_{1}^{\Lambda}\left[f\left(x^{5}\right)\right] & =\sum_{n=0}^{\infty} \frac{f^{(n)}(0)}{n !} * J_{1}^{(n) \Lambda}=f\left(x^{5}\right) d x^{5} \wedge \mathcal{G}_{2} \wedge \omega_{2}^{\Lambda}, \\
Q^{\Lambda}[f(t)] & =\int d^{5} x J^{0 \Lambda}[f(t)] \equiv \int_{M^{5}} * J_{1}^{\Lambda}[f(t)] \neq 0, \\
Q\left[f_{\Lambda}\left(x^{5}\right) \omega_{2}^{\Lambda}\right] & =\sum_{\Lambda} Q^{\Lambda}\left[f_{\Lambda}\left(x^{5}\right)\right] \neq 0 .
\end{aligned}
$$

In general the generating function for the Noether changes of semi-local symmetry (3.22) can be written as

$$
Q\left[\check{\varphi}_{2}\right]=\int_{M^{5}} d a(x) \wedge \mathcal{G}_{2} \wedge \check{\varphi}_{2}, \quad d \check{\varphi}_{2}=\check{\varphi}_{2}^{(1)} \wedge d a,
$$

where $M^{5}$ can be defined as a constant $t$ slice of $M^{1+5}$. In the $d a$-timelike branch of the dynamical system described by the PST action we can use the PST2 symmetry to fix the gauge $d a=d t$ in which it is immediate to see that $Q\left[\check{\varphi}_{2}\right]=0$. This is not the case for the $d a$-spacelike branches of PST system, where we can rather fix the gauge $d a=d x^{5}$ in which, generically, $Q\left[\check{\varphi}_{2}\right] \neq 0$.

Hence the semi-local symmetry is a gauge symmetry in the da-timelike branch of the dynamical system described by the PST action and an infinite dimensional rigid symmetry in the da-spacelike branch of the PST system.

\subsubsection{A speculation on possible alternative}

It is tempting, following the spirit of recent [33] (devoted to the Euclidean 5d SYM description of the mysterious non-Abelian $6 \mathrm{~d}(2,0)$ superconformal theory), to speculate on possible alternative canonical formalism allowing to treat the semilocal symmetry of PS action as a gauge symmetry.

Indeed, the above observation that the Noether current of semi-local symmetry of the PS action obeys $J^{5(n) \Lambda}=0$ (in particular cases, and $i_{5} * J_{1}\left[\left[\check{\varphi}_{2}\right]\right]=0$ in the general case) 
implies vanishing of 'pseudo-charge' constructed in the same way as Noether charge but with interchanging the role of time $x^{0}=t$ and the special space direction $x^{5}$,

$$
\tilde{Q}^{(n) \Lambda}:=\int d t d x^{1} d x^{2} d x^{3} d x^{4} J^{5(n) \Lambda}=0 .
$$

Then, following [33], one can build formally the canonical formalism based on using $x^{5}$ instead of time direction $x^{0}=t$. In its frame the semilocal symmetry of the PS action can be treated as a gauge symmetry and used to obtain the (anti-)self-duality equation. A necessary condition for the above treatment of PS action and of the $d a$-spacelike branch of the PST system in a topologically nontrivial spacetime with $b_{2} \neq 0$ is that this $M^{6}$ allows for the existence of a nowhere vanishing spacelike vector field, which can be associated with $x^{5}$ direction (besides the timelike nowhere vanishing vector field which is strictly necessary to have the metric of Lorentz signature). If so, one could state that also $d a$-spacelike branch of the covariant PST action, like $d a$-timelike one, can be used to obtain the antiself duality equation, provided certain alternative canonical formalism is used. The authors of [33] noticed that different choices of the basic variables of canonical formalism should correspond to restriction to a special field configurations for which the physically relevant integrals of the field variables (including the ones defining charges) do not diverge.

In this paper we will not elaborate the idea of alternative canonical formalism and keep the conservative point of view according to which the semilocal symmetry of the PS action and of the $d a$-spacelike branch of the PST system is an infinite dimensional rigid symmetry.

\section{4 (Anti-)self-duality equation from PST action in topologically nontrivial $M^{6}$}

Thus, as we have shown above, in the topologically nontrivial space-time $M^{1+5}$ the PST action produces the dynamical equations of motion (2.21), which are equivalent to (3.19). In particular cases of topologically nontrivial spacetimes this first order form of the Lagrangian equations can be written in a more transparent manner (3.20),

$$
\mathcal{G}_{2} \wedge d a:=i_{v}\left(H_{3}+* H_{3}\right) \wedge v=l_{\Lambda}(a) \omega^{\Lambda} \wedge d a
$$

in which the r.h.s. is expressed in terms of $b_{2}$ arbitrary functions of the PST scalar $l_{\Lambda}=$ $l_{\Lambda}(a(x))$. Below we will carry the discussion in terms of this particular case and give the generic form of the equations in parenthesis. (See (3.19) for the first order form of the PST Lagrangian equations in a generic spacetime).

On the other hand the PST action possesses, in addition to the PST1 and PST2 gauge symmetries, also the semi-local symmetry which in these particular spacetimes, is parametrized by $b_{2}$ arbitrary functions of the PST scalar $f_{\Lambda}(a(x))$ in (3.23),

$$
\delta B_{2}=\varphi_{1} \wedge d a+\delta a \mathcal{G}_{2}+\omega_{2}^{\Lambda} f_{\Lambda}(a(x))
$$

((3.22) in the generic case). 


\subsection{1. $d a$-timelike branch}

In the da-timelike branch of the PST system, described by the PST action with timelike $\partial_{\mu} a$, all these are gauge symmetries and one can use them to gauge away the r.h.s. of (3.41), thus arriving at $\mathcal{G}_{2} \wedge d a:=i_{v}\left(H_{3}+* H_{3}\right) \wedge v=0$ which is equivalent to the anti-self-duality (2.29),

$$
H_{3}+* H_{3}=0
$$

for the 'original' field strength (2.8) entering the action (2.11).

\subsection{2. $d a$-spacelike branch}

As we have already discussed in section 3.3.1, to treat similarly the da-spacelike branch of the PST system, as well as the PS system appearing as the gauge fixed, $d a=d x^{5}$ version of this branch, one might try, following the spirit of [33] to develop an alternative canonical formalism using $x^{5}$ variable instead of time.

In this paper we will follow a more conservative approach keeping the standard relation of canonical formalism with the timelike direction of $M^{1+5}$. Then, the semi-local symmetry of the da-spacelike branch of the PST system is an infinite dimensional global symmetry, which cannot be used to fix a gauge, and the Lagrangian equation (2.21) is gauge equivalent to

$$
\mathcal{G}_{2} \wedge d a:=i_{v}\left(H_{3}+* H_{3}\right) \wedge v=l_{\Lambda}(a) \omega^{\Lambda} \wedge d a
$$

with arbitrary functions of the PST scalar $l_{\Lambda}(a)$ (to (3.19) with $\phi_{1}=0$ in the general case).

Thus the standard anti-self-duality equation (3.43) for the field strength $\mathrm{H}_{3}$ entering the action cannot be reproduced in the da-spacelike branch. However, let us observe that we can remove the additional topological contributions by redefining the field strength as

$$
\tilde{H}_{3}=H_{3}-l_{\Lambda}(a(x)) \omega^{\Lambda} \wedge d a(x)
$$

(or, in the general case,

$$
\tilde{H}_{3}=H_{3}-\check{\omega}_{2} \wedge d a(x), \quad d \check{\omega}_{2}=\check{\omega}_{2}^{(1)} \wedge d a(x),
$$

with $\check{\omega}_{2}$ being arbitrary in all other respects). Indeed, due to (3.44) (or (3.19)), such $\tilde{H}_{3}$ obeys, besides the standard Bianchi identities $d \tilde{H}_{3}=0$, also $i_{v}\left(\tilde{H}_{3}+* \tilde{H}_{3}\right)=0$ and hence

$$
\tilde{H}_{3}+* \tilde{H}_{3}=0 \text {. }
$$

Actually, when we can consider $d a(x)$ (and hence $d a(x) l_{\Lambda}(a)$ in (3.45)) as an exact form, we can write the redefinition of the field strength as a redefinition of the potential,

$$
H_{3}=d B_{2}, \quad \tilde{H}_{3}=d \tilde{B}_{2}, \quad \tilde{B}_{2}=B_{2}+\omega_{2}^{\Lambda} \tilde{f}_{\Lambda}(a)+d \alpha_{1}, \quad \frac{d}{d a} \tilde{f}_{\Lambda}(a)=l_{\Lambda}(a)
$$

$\left(\tilde{B}_{2}=B_{2}+\beta_{2}\right.$ with $d \beta_{2}=\check{\omega}_{2} \wedge d a$ in general case).

This allows us to state that, in a topologically nontrivial spacetime with $b_{2} \neq 0$, and more generally in the spacetime which allows for an existence of a nontrivial solution 
$\left(\check{\omega}_{2} \neq d \chi_{1}+i_{v} d \chi_{1} \wedge v\right)$ of the equation $d \check{\omega}_{2}=\check{\omega}_{2}^{(1)} \wedge d a$, the $d a$-spacelike branch of the PST system, as well as the non-manifestly covariant PS action, can be used to produce the (anti-)self-duality equation only for the redefined field strength $\tilde{H}_{3}(3.47)$ or redefined potential $\tilde{B}_{2}$ (3.48). In particular cases where the redefinition has an especially transparent form, it involves $b_{2}$ arbitrary functions of the PST scalar (of a special spacial coordinate $x^{5}$ in the case of PS action) which can be associated with the set of parameters of semilocal symmetry. However, as far as this symmetry is an infinite dimensional global symmetry rather than a gauge symmetry, the original and redefined potentials, $B_{2}$ and $\tilde{B}_{2}$, cannot be considered as equivalent in this case.

Thus the topology makes difference between $d a$-timelike and $d a$-spacelike branches of the PST system as well as between non-manifestly invariant HT and PS actions. The timelike branch of the PST system and its gauge fixed version described by HT action, become preferable as they produce the (anti-)self-duality equation for the original field strength (field strength of the original potential), which enters the action, also in spacetime with $b_{2} \neq 0$ and more generally in spacetime allowing for existence of a nontrivial solution $\left(\check{\omega}_{2} \neq d \chi_{1}-i_{v} d \chi_{1} \wedge v\right)$ of the equation $d \check{\omega}_{2}=\check{\omega}_{2}^{(1)} \wedge d a$.

\subsubsection{On spacetime of Euclidean signature $M^{6}=M^{6+0}$}

In the case of topologically nontrivial spacetime of Euclidean signature, $M^{6}=M^{6+0}$, the main problem is that, to obey $*^{2}=1$, the Hodge duality operation is to be imaginary, $\left(* H_{3}\right)^{*}=-*\left(H_{3}\right)^{*}$ so that the (anti-)self-dual gauge field should be complex, $\left(H_{3}\right)^{*} \neq H_{3}$. However, if we allow for complex fields and non-Hermitian actions (this is widely used e.g. in pure spinor approach to quantum superstring [34, 35]; see also [10]), then the only requirement for the spacetime $M^{6}=M^{6+0}$ to define (a complex) PST action is to allow for a nowhere vanishing vector fields. Then if such field is unique, the only possibility is to identify $d a$ and $d t$ with it(s dual) and the PST action automatically reduces to HT one.

In $M^{6}=M^{6+0}$ of Euclidean signature with several nowhere vanishing vector fields all the allowed choices of $d a$ can be related by a nonsingular PST2 gauge transformations so that no separations on the branches in (complex) PST action occurs and the (complex) HT and PS actions are gauge equivalent. In some particular cases, including $M^{6}$ with $b_{2}\left(M^{6}\right) \neq 0$, the additional contributions to the r.h.s. of the first order form of the Lagrangian equation which follows from the PST action do appear; however the PST action possesses the semi-local symmetry, and this can be treated as gauge symmetry. Hence the (complex) PST action in such an $M^{6}=M^{6+0}$ produces the Lagrangian equations which are gauge equivalent to the anti-self-duality conditions (2.29) for the original (complex) gauge field strength which enters the action.

\subsection{Summarizing the case of chiral 2 -form gauge potential in 6 dimensions}

Thus we have shown that the PST action for the 6D 2-form potential can be used to obtain the self-duality equations also in the topologically nontrivial spacetime.

Interestingly enough, the $d a$-timelike and $d a$-spacelike branches of the PST system become nonequivalent in the spacetime $M^{1+5}$ with $b_{2}=\operatorname{dim} \mathbb{H}^{2}\left(M^{1+5}\right) \neq 0$ or, more generally if $M^{1+5}$ allows for a nontrivial solution $\left(\check{\omega}_{2} \neq d \chi_{1} \mp i_{v} d \chi_{1} \wedge v\right)$ of the equation 
$d \check{\omega}_{2}=\check{\omega}_{2}^{(1)} \wedge d a$ with some closed 1-form $d a=d a(x)(v \propto d a)$. Also the non-manifestly invariant HT and PS actions, which can be obtained from these branches of PST system by gauge fixing of PST2 symmetry, are non-equivalent in such spacetimes. In both cases the Lagrangian equations are equivalent to the (anti-)self-duality conditions for redefined field strength and the redefinition can be identified with some semi-local symmetry of the PST action. However, the $d a$-timelike PST system is preferable as in it the semi-local symmetry is a gauge symmetry so that the redefined field strength is gauge equivalent to the original one which enters the action. Thus, in this $d a$-timelike branch of PST system (and in the HT action) the Lagrangian equations are equivalent to the anti-self-duality equation for the original field strength entering the PST action.

Similar situation occurs for the chiral $2 l$-form gauge field in topologically nontrivial spacetime of $D=4 l+2$ dimensions and, with a minimal modification, also for any even $D$. But before describing this general case, we turn to the simplest $\mathrm{D}=2$ chiral boson system, in which a semi-local symmetry occurs also in PST action in the topologically trivial $\mathrm{D}=2$ spacetime.

\section{Prototype of the topological gauge symmetry in 2D PST action for chiral bosons}

The simplest, but also special case of theories of self-dual and anti-self-dual tensor fields is the theory of chiral boson in two dimensional spacetime. In a topologically trivial spacetime one can fix the conformal gauge, where the chiral (anti-chiral) bosons are represented by functions of only $t-x$ (only $t+x) .{ }^{6}$ In terms of differential forms, one defines the 2 d Hodge star operation by

$$
\begin{aligned}
* d \phi & =*\left(d x^{m} \partial_{m} \Phi\right)=d x^{m} \sqrt{|g|} \epsilon_{m n} g^{n k} \partial_{k} \phi, \\
* 1 & =d^{2} x \sqrt{|g|}:=\frac{1}{2} d x^{m} \wedge d x^{n} \epsilon_{n m} \sqrt{|g|}, \quad * *=I,
\end{aligned}
$$

with $\epsilon_{m n}=-\epsilon_{n m}, \epsilon^{01}=-\epsilon_{01}=1$, and writes the chirality condition

$$
\left(\partial_{t}+\partial_{x}\right) \phi=0
$$

as $2 \mathrm{~d}$ anti-self-duality equation,

$$
d \phi+* d \phi=0 .
$$

The Lagrangian 2-form of 2D PST action can be written as

$$
\mathcal{L}_{2}=i_{v}(d \phi+* d \phi) d \phi \wedge v=\frac{1}{2 v^{2}} d \phi \wedge * d \phi-\frac{1}{2} i_{v}(d \phi+* d \phi) * i_{v}(d \phi+* d \phi) .
$$

For completeness of this section let us recall that $v=d x^{m} v_{m}$ is defined in (2.12), $v^{2}= \pm 1$, and $i_{v}$ - by $i_{v} d \phi=v^{m} \partial_{m} \phi$ and by the 2-form counterpart of (2.13). Denoting $F=d \phi$, we can write the variation of the Lagrangian form (4.4) as

$$
\delta \mathcal{L}_{2}=\delta F \wedge v i_{v}(F+* F)-\delta v \wedge v\left(i_{v}(F+* F)\right)^{2}+\frac{1}{2 v^{2}} F \wedge \delta F
$$

\footnotetext{
${ }^{6}$ In this section we use the notation $x^{m}=\left(x^{0}, x^{1}\right)=(t, x)$, so that $f(x)$ denotes the function of one rather than of two coordinates.
} 
and, ignoring the total derivatives, as

$$
\delta \mathcal{L}_{2}=d a \wedge d \mathcal{G}_{0}\left(\delta \phi-2 \delta a \mathcal{G}_{0}\right)
$$

Here

$$
\mathcal{G}_{0}=\frac{i_{v}(F+* F)}{\sqrt{\partial a \partial a}}=\frac{i_{v}(d \phi+* d \phi)}{\sqrt{\partial a \partial a}} .
$$

From (4.6) one can clearly read the second PST symmetry with $\delta \phi=2 \delta a \mathcal{G}_{0}$ and arbitrary function $\delta a(t, x)$. But, as far as our basic field is now scalar, the straightforward counterpart of the first PST symmetry $\left(\delta B_{p}=\phi_{p-1} \wedge d a\right.$, see the first term in (3.23)) is actually absent.

A more careful analysis shows that $\delta \phi=f(a(t, x))$ gives only a total derivative contribution to (4.6) so that the complete symmetry variation leaving invariant the PST action for the chiral bosons reads

$$
\delta \phi=2 \delta a \mathcal{G}_{0}+f(a(t, x)) .
$$

On one hand, as the function $f(a(t, x))$ depends on the 2 d spacetime coordinates through its dependence on the PST scalar only, the second term is the clear counterpart of the semi-local symmetry of the above section 3.2. On the other hand this symmetry is present in the action for chiral bosons in a topologically trivial spacetime as well, and, as we will see below, plays the role of PST1 symmetry. This is why we would like to call it semi-local PST1 symmetry. Let us study how it works in the derivation of the chirality (anti-self-duality) equation (4.3) in topologically trivial spacetime $M^{1+1}$.

Eq. (4.6) makes transparent that the equation of motion which follow from PST action with (4.4) reads

$$
d a \wedge d \mathcal{G}_{0} \equiv d\left(d a \mathcal{G}_{0}\right)=0 .
$$

In the topologically trivial situation $\left(b_{1}=\operatorname{dim} \mathbb{H}^{1}\left(M^{1+1}\right)=0\right)$ it is solved by

$$
\mathcal{G}_{0}=\tilde{f}(a(t, x)),
$$

where $\tilde{f}(a(t, x))$ is an arbitrary function of the PST scalar $a(t, x)$.

Now we observe that under the semilocal PST1 symmetry (4.8)

$$
\delta \mathcal{G}_{0}=f^{\prime}(a(t, x)),
$$

so that r.h.s. of (4.10) can be removed or generated by the transformation with $f^{\prime}(a):=$ $\frac{d}{d a} f=\mp \tilde{f}(a)$. Then, if the semilocal PST1 symmetry is a gauge symmetry, eq. (4.10) is gauge equivalent to $\mathcal{G}_{0}=0$ which in its turn is tantamount to the chirality equation (4.3),

$$
\mathcal{G}_{0}=0 \quad \Leftrightarrow \quad d \phi+* d \phi=0 .
$$

As we will see, this is the case for da-timelike branch of the dynamical system described by the PST action (4.4) (da-timelike PST) which is gauge equivalent to the not manifestly Lorentz invariant Floreanini-Jackiw (FJ) action [20],

$$
S_{F J}=-\int d t d x\left(\partial_{t} \phi \partial_{x} \phi+\partial_{x} \phi \partial_{x} \phi\right) .
$$


In contrast, for the $d a$-spacelike branch of the $2 \mathrm{~d}$ PST system (da-spacelike PST), which is gauge equivalent to non-manifestly Lorentz invariant 'anti-FJ' or PS-like action,

$$
S_{a F J}=\int d t d x\left(\partial_{t} \phi \partial_{t} \phi+\partial_{t} \phi \partial_{x} \phi\right)
$$

the semi-local PST1 is an infinite dimensional global symmetry.

\subsection{Semi-local symmetry as gauge symmetry of FJ and $d a$-timelike PST ac- tions}

Using the PST2 symmetry of the $d a$-timelike PST system to fix the gauge $a(t, x)=t$, where the PST action is reduced to the FJ action (4.13), we notice that the $2 \mathrm{~d}$ counterpart of the PST1 symmetry, the semi-local PST1 symmetry, is parametrized by a function of time coordinate $x^{0}=t$ only,

$$
\delta \phi(t, x)=f(t)
$$

Notice that, if the spacial coordinate takes values in a final interval, $x \in\left(x_{f}, x_{i}\right),(4.15)$ is also the symmetry of the action provided the scalar field $\phi(t, x)$ obeys the following boundary conditions

$$
\phi\left(t, x_{f}\right)=\phi\left(t, x_{i}\right) .
$$

The fact that (4.15) is a gauge symmetry is intuitively clear. However, it is instructive to prove this formally.

As it was stressed in recent [30] (see also [31, 32]) the difference between gauge symmetry and infinite dimensional global symmetry, the characteristic example of which is given by the $2 \mathrm{~d}$ conformal symmetry, is that for the former the Noether charges vanish identically, while for the latter this is not the case.

Decomposing the parametric function on (4.15) in series, $f(t)=f(0)+t f^{\prime}(0)+\ldots+$ $\frac{t^{n}}{n !} f^{(n)}(0)+\ldots$, and considering $\epsilon^{(n)}=\frac{f^{(n)}(0)}{n !}$ as the symmetry parameters, we find the corresponding Noether currents $J^{\mu(n)}=\left(J^{0(n)}, J^{1(n)}\right)$,

$$
\begin{aligned}
& J^{0(n)}=-t^{n} \partial_{x} \phi \equiv-\partial_{x}\left(t^{n} \phi\right), \\
& J^{1(n)}=-t^{n}\left(\partial_{t}+2 \partial_{x}\right) \phi+n t^{n-1} \phi \equiv \partial_{t}\left(t^{n} \phi\right)-2 t^{n}\left(\partial_{t}+\partial_{x}\right) \phi .
\end{aligned}
$$

It is not difficult to see that these currents are conserved on the mass shell, $\partial_{\mu} J^{\mu(n)}=$ $\partial_{t} J^{0(n)}+\partial_{x} J^{1(n)}=-2 t^{n} \partial_{x}\left(\partial_{t}+\partial_{x}\right) \phi$, and that the corresponding Noether charges

$$
Q^{(n)}=\int d x J^{0(n)}=\int d x \partial_{x}\left(t^{n} \phi\right)=-t^{n}\left(\phi\left(t, x_{f}\right)-\phi\left(t, x_{i}\right)\right)=0
$$

vanish identically with the boundary conditions (4.16).

Hence, the semilocal PST1 symmetry of the FJ action (4.13) is gauge symmetry. The same conclusion holds for the semi-local PST1 symmetry of the $d a$-timelike branch of the dynamical system described by the manifestly Lorentz invariant 2d PST action (4.4). 


\subsection{Chirality equation as gauge fixed form of the Lagrangian equations of the FJ action and of the da-timelike branch of the PST action}

The Lagrangian equation of motion which follows from the FJ action (4.13), $\partial_{x}\left(\partial_{t}+\partial_{x}\right) \phi=$ 0 , can be written in the first order form as

$$
\left(\partial_{t}+\partial_{x}\right) \phi=\varphi(t) .
$$

However, the action (4.13) and the second order form of the equations are invariant under the semi-local PST1 symmetry (4.15) which, as we have shown above, is a gauge symmetry. Choosing $f(t)$ in (4.15) to be a solution of $\dot{f}(t):=\partial_{t} f(t)=\varphi(t)$ we can gauge away the r.h.s. of (4.19) and write this equation in a gauge fixed form

$$
\left(\partial_{t}+\partial_{x}\right) \phi=0 \text {. }
$$

Hence the FJ action (4.13) can be used to obtain the chirality condition (4.20) as equation of motion.

This is also the case for the $d a$-timelike branch of the PST system. The Lagrangian equations (4.9) which follow from the PST action (4.4) are gauge equivalent to the chirality conditions (4.12), $d \phi+* d \phi=0$, as the semi-local PST1 symmetry is a gauge symmetry in this branch.

\subsection{Issues of anti-FJ action and $d a$-spacelike branch of the 2d PST system}

In the case of anti-FJ action (4.14) which can be obtained from $d a$-spacelike branch of the PST system by gauge fixing of the PST2 symmetry, the semi-local PST1 symmetry transformation are characterized by a function of spacial variable $x$,

$$
\delta \phi(t, x)=f(x) .
$$

A more careful look shows that this symmetry requires the 'initial' conditions

$$
\phi\left(t_{i}, x\right)=\phi\left(t_{f}, x\right)
$$

(in contrast with the boundary conditions (4.16) required for the semi-local symmetry of the FJ action). The components of Noether currents $J^{\mu(n)}=\left(J^{0(n)}, J^{1(n)}\right)$ corresponding to (infinitely many constant parameters of) this symmetry are

$$
J^{0(n)}=2 x^{n}\left(\partial_{t}+\partial_{x}\right) \phi-\partial_{x}\left(x^{n} \phi\right), \quad J^{1(n)}=\partial_{t}\left(x^{n} \phi\right) .
$$

These currents are conserved on the mass shell and have the Noether charges

$$
Q^{(n)}=\int d x J^{0(n)}=-\int d x \partial_{x}\left(x^{n} \phi\right)=x_{i}^{n} \phi\left(t, x_{i}\right)-x_{f}^{n} \phi\left(t, x_{f}\right) \neq 0 .
$$

Generically, these do not vanish. Hence we conclude that the semi-local PST1 symmetry of the anti-FJ action (4.14) and of the $d a$-spacelike branch of the $2 \mathrm{~d}$ PST system is not a gauge symmetry but an infinite dimensional rigid symmetry, similar to the famous $2 \mathrm{~d}$ conformal symmetry. 
That infinite dimensional rigid symmetry cannot be used to gauge away the r.h.s. of the first order form of the anti-FJ Lagrangian equation which, thus, contains an arbitrary function of the spacial coordinate $x$ in r.h.s.,

$$
\left(\partial_{t}+\partial_{x}\right) \phi=\varphi(x)
$$

Of course, this can be written as a chirality equation for the redefined field $\tilde{\phi}=\phi(t, x)-$ $\varphi(x)$

$$
\left(\partial_{t}+\partial_{x}\right) \tilde{\phi}=0, \quad \tilde{\phi}=\phi(t, x)-\varphi(x) .
$$

However, this does not help much as far as the redefinition is done with an arbitrary function and it does not change the conclusion that the general solution of the equations of motion which follow from the anti-FJ action,

$$
\phi(t, x)=h(t-x)-\varphi(x),
$$

contains, besides the arbitrary chiral function $h(t-x)$, also arbitrary function of the spacial variable $\varphi(x)$.

Thus in the case of $2 \mathrm{~d}$ chiral bosons, even when $2 \mathrm{~d}$ spacetime is topologically trivial, the $d a$-timelike branch of the $2 \mathrm{~d}$ PST system and a non-manifestly Lorentz invariant FJ action, which can be obtained from that by gauge fixing, are preferable over the $d a$-spacelike branch of the 2d PST system and anti-FJ action. Only formers can be used to obtain the anti-self duality equations, (4.12) and (4.20), the general solution of which are given by one arbitrary function $h(t-x)$. In contrast, for $d a$-spacelike PST and anti-FJ action the general solution of the equations of motion, eq. (4.27), contains also an arbitrary function of the spacial coordinate, $\varphi(x)$, and in this sense is rather similar to the solution of the equations of motion of 'usual', non-chiral massless boson.

\subsection{A speculation on alternative canonical formalism in $2 \mathrm{~d}$}

In search for possibility to rehabilitate the $d a$-spacelike branch of the PST system and the anti-FJ action one may turn to the idea of [33] to develop the canonical formalism using $x$ instead of $t$ variable.

Indeed, the pseudo-Noether-charge assiciated to this formalism,

$$
\tilde{Q}^{(n)}=\int d t J^{1(n)}=\int d t \partial_{t}\left(x^{n} \phi\right)=x^{n}\left(\phi\left(t_{f}, x\right)-\phi\left(t_{i}, x\right)\right)=0
$$

vanishes identically as a result of 'initial' conditions (4.22). As it was noticed in [33], different choices of the basic variable of the canonical formalism should correspond to restrictions to different field configurations, for which the different physically relevant integrals converge. In the two-dimensional case spacial and temporal slices are one-dimensional so that the exchange of the roles of space and time variables, and the convergence conditions for spacial and temporal integrals, does not look unnatural.

However, after fixing once the basic variable (time) of canonical formalism, one sees that the dynamical system described by the PST action splits on two branches, $d a$-timelike 
and $d a$-spacelike, and that the semi-local PST symmetry is the gauge symmetry in one, usually chosen to be the first, while it is the infinite dimensional rigid symmetry in the other. Then the $d a$-timelike branch, the gauge fixed version of which are given by nonmanifestly Lorentz invariant FJ action, is preferable as it allows to obtain the chiral boson equation as a gauge fixed version of the Lagrangian equation of motion.

\subsection{Chiral bosons on a Riemann surface}

On Riemann surface $\Sigma_{g}$ with nonvanishing genus $\mathbf{g} \neq 0$ the first cohomology group $\mathbb{H}^{1}\left(\Sigma_{g}\right)$ is $2 \mathrm{~g}$ dimensional, $b_{1}=2 \mathrm{~g} \neq 0$, so that there exists a basis $\left\{\Omega_{1}^{L}\right\}$ of $2 \mathrm{~g}$ closed but not exact forms on $\Sigma_{g}$. If $\Sigma_{g}$ allows for the existence of a nowhere vanishing vector field, the PST action (4.4) is well defined and produces the Lagrangian equation (4.9). ${ }^{7}$ On $\Sigma_{g}$ the general solution of (4.9) reads

$$
d a \mathcal{G}_{0}=d a \tilde{f}(a)+k_{L} \Omega_{1}^{L}, \quad d \Omega_{1}^{L}=0, \quad \Omega_{1}^{L} \neq d \chi(x), \quad L=1, \ldots, 2 \mathbf{g} .
$$

It includes $2 \mathrm{~g}$ constants $k_{L}$.

Contracting this equation with $v=d a / \sqrt{|\partial a \partial a|}$, we find

$$
\mathcal{G}_{0}=\tilde{f}(a) \pm k_{L} i_{v} \Omega_{1}^{L} / \sqrt{|\partial a \partial a|}
$$

while contracting the Hodge dual of eq. (4.29) gives the following equation for the coefficients $k_{L}$ :

$$
k_{L} i_{v} * \Omega_{1}^{L}=0
$$

Similar equations can be derived from non-manifestly diffeomorphism invariant FJtype action obtained from the da-timelike branch of PST action by setting $d a=d t$. These read

$$
\begin{array}{cl}
\mathcal{G}_{0}:=i_{0}(d \phi+* d \phi)= & \tilde{f}(t)+k_{L} i_{0} \Omega_{1}^{L}, \\
k_{L} i_{0} * \Omega_{1}^{L}=0 & \Leftrightarrow \quad k_{L} i_{1} \Omega_{1}^{L}=0 .
\end{array}
$$

The last equation restricts the set of constants $k_{L}$ to be such that $k_{L} \Omega_{1}^{L}=d t k_{L} i_{0} \Omega_{1}^{L}$. Then the closure of $\Omega_{1}^{L}$ forms implies $d\left(k_{L} \Omega_{1}^{L}\right)=d t \wedge d\left(k_{L} i_{0} \Omega_{1}^{L}\right)=0$ and hence that $k_{L} i_{0} \Omega_{1}^{L}$ is $x$-independent, $k_{L} i_{0} \Omega_{1}^{L}=\tilde{\tilde{f}}(t){ }^{8}$ As a result, eq. (4.32) can be written in the form

$$
\mathcal{G}_{0}:=i_{0}(d \phi+* d \phi)=\tilde{f}(t)+\tilde{\tilde{f}}(t) .
$$

\footnotetext{
${ }^{7}$ It is known that if the Riemann surface is compact, connected and admits a nowhere vanishing holomorphic one-form $((1,0)$-form), then it is a torus (quotient of $\mathbb{C}$ by a lattice) which implies $\mathbf{g}=1$. An exhaustive study of the canonical and BRST quantization of the FJ model on the torus and comparison of the particion function for chiral bosons obtained on this way with the results of holomorphic factorization approach [36] can be found in recent [37].

${ }^{8}$ Then the form $k_{L} \Omega_{1}^{L}=d t \tilde{\tilde{f}}(t)$ is not exact iff $d t$ is not exact, i.e. iff our time is an angular variable. If not, then the condition that $\Omega_{1}^{L}$ forms are not exact would require to set $\tilde{\tilde{f}}(t)=0$ and we can proceed as in the topologically trivial case.
} 
Then the second term together with the first one can be gauged away using the semilocal PST1 symmetry (4.15) with $f(t)$ obeying $f^{\prime}(t)=-\tilde{f}(t)-\tilde{\tilde{f}}(t)$. After this stage we arrive at the chirality equation (4.3).

In the same manner we can reproduce the chirality equation (4.3) as a gauge fixed version of the Lagrangian equations of motion which follow from the PST action (4.4) written on a Riemann surface $\Sigma_{g}$, but only in its da-timelike branch in which the semilocal PST1 symmetry in (4.8) is a gauge symmetry (see however the speculations in the previous subsection). ${ }^{9}$

\section{Twisted anti-self-duality of p-form gauge fields from the PST action in a $\mathrm{D}=2 \mathrm{p}+2$ dimensional spacetime of nontrivial topology}

The generalization of the analysis of section 3 to chiral bosons in spacetime of an arbitrary even dimension is quite straightforward. In this section we will present the basic equations and formulate the conclusions for the Lagrangian description of chiral p-form gauge fields in topologically nontrivial spacetime of $D=2 p+2$ dimensions.

\subsection{Twisted anti-self-duality in $D=2 p+2$}

Let us define the measure of $D=2 p+2$ dimensional spacetime by $d x^{\nu_{1}} \wedge \ldots \wedge d x^{\nu_{D}}=$ $d^{D} x \epsilon^{\nu_{1} \ldots \nu_{D}}=-(-)^{p} d^{D} x \epsilon^{\nu_{D} \ldots \nu_{1}}$ with $\epsilon^{01 \ldots(2 p+1)}=-\epsilon_{01 \ldots(2 p+1)}=1$, and consider the set of $n p$-form gauge fields $B_{p}^{I}, I=1, \ldots, n$, with the field strength (see also (5.19) below)

$$
\begin{array}{r}
H_{p+1}^{I}=d B_{p}^{I}=\frac{1}{(p+1) !} d x^{\nu_{p+1}} \wedge \ldots \\
\nu=0,1, \ldots,(2 p+1) .
\end{array}
$$

If we define the dual of an arbitrary $q$-form $F_{q}=\frac{1}{q !} d x^{\nu_{q}} \wedge \ldots \wedge d x^{\nu_{1}} F_{\nu_{1} \ldots \nu_{q}}(x)$ by

$$
* F_{q}=\frac{1}{(D-q) !} d x^{\nu_{D-q}} \wedge \ldots \wedge d x^{\nu_{1}} \frac{\sqrt{|g|}}{q !} \epsilon_{\nu_{1} \ldots \nu_{D-1} \mu_{1} \ldots \mu_{q}} F^{\mu_{1} \ldots \mu_{q}}(x)
$$

then $* * H_{p+1}^{I}=(-)^{p} H_{p+1}^{I}$ so that for odd $p$ (in particular for $p=1$ corresponding to $D=4$ ) to be consistent one has to consider a twisted (anti-)self-duality condition imposed on the even number of gauge field strengths $(n=2 m$ for $p=2 q+1)$, rather than just (anti-)self-duality condition. The twisted anti-self-duality equation has the form

$$
\mathcal{H}_{p+1}^{I}=0
$$

where

$$
\mathcal{H}_{p+1}^{I}=H_{p+1}^{I}+\Omega_{I J} * H_{p+1}^{J} .
$$

\footnotetext{
${ }^{9}$ If $\Sigma_{g}$ has the metric of Euclidean signature, then there is no separation of PST system on two branches, all the possible values of $d a$ are related by nonsingular PST2 symmetry transformations, but the chiral boson and the PST Lagrangian become complex. See section 3.5.3 for more discussion in the $D=6$ model.
} 
This contains an invertible $n \times n$ matrix $\Omega_{I J}$ with the properties

$$
\Omega_{I J}=(-)^{p} \Omega_{J I}, \quad \Omega_{I K} \Omega_{K J}=(-)^{p} \delta_{I J}, \quad I, J=1, \ldots, n .
$$

As a result,

$$
\mathcal{H}_{p+1}^{I} \equiv \Omega_{I J} * \mathcal{H}_{p+1}^{J}
$$

and

$$
\Omega_{I J} H_{p+1}^{I} \wedge H_{p+1}^{J}=0
$$

hold.

\subsection{PST action for chiral bosons in $M^{2 p+2}$}

When spacetime $M^{D}=M^{1+(2 p+1)}$ is topologically trivial, it is known that the twisted anti-self duality equation (5.3) can be obtained from the PST action $S^{P S T} \propto \int \mathcal{L}_{D}^{P S T}$ with

$$
\begin{gathered}
\mathcal{L}_{D}^{P S T}=-\Omega_{I J} i_{v} \mathcal{H}_{p+1}^{I} \wedge H_{p+1}^{J} \wedge v=\frac{1}{p !} d^{D} x \sqrt{|g|} v^{\rho} \mathcal{H}_{\rho \mu_{1} \ldots \mu_{p}}^{I} v_{\lambda} * H^{\lambda \mu_{1} \ldots \mu_{p}} \\
v=d x^{\nu} v_{\nu}=d a / \sqrt{|\partial a \partial a|}, \quad \partial a \partial a:=g^{\mu \nu} \partial_{\mu} a \partial_{\nu} a
\end{gathered}
$$

Indeed, modulo exact forms, the variation of $\mathcal{L}_{D}^{P S T}$ can be written as ${ }^{10}$

$$
\delta \mathcal{L}_{D}^{P S T}=2(-)^{p} \Omega_{I J} d\left(d a \wedge \mathcal{G}_{p}^{I}\right) \wedge\left(\delta B_{p}^{J}-\delta a \mathcal{G}_{p}^{J}\right),
$$

where

$$
\mathcal{G}_{p}^{I}:=\frac{i_{v} \mathcal{H}_{p+1}^{I}}{\sqrt{|\partial a \partial a|}}=\frac{i_{v} H_{p+1}^{I}+\Omega_{I J} i_{v} * H_{p+1}^{J}}{\sqrt{|\partial a \partial a|}} .
$$

Eq. (5.10) makes manifest the PST gauge symmetries

$$
\delta B_{p}^{I}=\varphi_{p-1}^{I} \wedge d a+\delta a \mathcal{G}_{p}^{I}
$$

with an arbitrary $\delta a(x)$ and $\varphi_{\mu_{1} \ldots \mu_{p-1}}^{I}(x)=\varphi_{\left[\mu_{1} \ldots \mu_{p-1}\right]}^{I}(x)$ in $\varphi_{p-1}^{I}=\frac{1}{(p-1) !} d x^{\mu_{p-1}} \wedge$ $\ldots d x^{\mu_{1}} \varphi_{\mu_{1} \ldots \mu_{p-1}}^{I}(x)$. The presence of the former shows the pure gauge (Stückelberg) nature of the PST scalar, while the arbitrary $\varphi_{\mu_{1} \ldots \mu_{p-1}}^{I}(x)$ allows to gauge away the general solution $d a \wedge \mathcal{G}_{p}=d a \wedge d \phi_{p-1}$ of the Lagrangian equations of motion

$$
d\left(d a \wedge \mathcal{G}_{p}^{I}\right)=0
$$

${ }^{10}$ In terms of the variation of the field strength

$$
\delta \mathcal{L}_{D}^{P S T}=2 \Omega_{I J} d a \wedge \mathcal{G}_{p}^{I} \wedge\left(\delta H_{p+1}^{J}-\frac{1}{2} \mathcal{G}_{p}^{J} \wedge d(\delta a)\right) \mp(-)^{p} \Omega_{I J} H_{p+1}^{I} \wedge \delta H_{p+1}^{J},
$$

where the sign of the last term is related to $\pm 1=v_{\mu} v^{\mu}$. The property (5.7) is essential to derive this result. The other useful identities are $\pm F_{q}=i_{v} F_{q} \wedge v+*\left(i_{v} * F_{q} \wedge v\right)$ and $i_{v} * F_{q}=-(-)^{q} *\left(F_{q} \wedge v\right)$, in particular, $i_{v} * H_{p+1}=(-)^{p} *\left(H_{p+1} \wedge v\right)$. 
thus arriving at $\mathcal{G}_{p}^{I}=0$ which implies the twisted anti-self-duality equation (5.3)

$$
\mathcal{G}_{p}^{I}=0 \quad \Rightarrow \quad \mathcal{H}_{p}^{I}=0 .
$$

Like in $D=6$, for our discussion below we need to define da-timelike and da-spacelike branches, in which the partial derivative of the PST scalar is spacelike and timelike vector respectively:

$$
\begin{aligned}
d a-\text { timelike branch }: & \partial а \partial a:=g^{\mu \nu} \partial_{\mu} a \partial_{\nu} a>0, \\
d a-\text { spacelike branch }: & \partial a \partial a:=g^{\mu \nu} \partial_{\mu} a \partial_{\nu} a<0 .
\end{aligned}
$$

In the da-timelike branch, the PST scalar $a(x)$ can be equated to the time coordinate by using the smooth local PST2 transformation, while in the da-spacelike branch this is impossible but it is possible to equate $a(x)$ with one of the space coordinates, say $d a(x)=$ $d x^{1}$. Indeed, these two choices, $d a(x)=d x^{0}$ and $d a(x)=d x^{1}$, clearly corresponding to (5.15) and (5.16), cannot be related by smooth local PST2 transformations (see section 2.2.3 for more details).

\subsection{Twisted anti-self-duality from PST action in topologically nontrivial $M^{2 p+2}$}

In the topologically nontrivial spacetime $M^{2 p+2}=M^{1+(2 p+1)}$ with nonvanishing Bette numbers $b_{p}=\operatorname{dim} \mathbb{H}^{p}\left(M^{2 p+2}\right) \neq 0$ and $b_{p+1}=\operatorname{dim} \mathbb{H}^{p+1}\left(M^{2 p+2}\right) \neq 0$ there exist $b_{p}$ closed but not exact $p$-forms $\omega_{p}^{\Lambda}$ which provide the basis of $\mathbb{H}^{p}\left(M^{2 p+2}\right)$,

$$
d \omega_{p}^{\Lambda}=0, \quad \omega_{p}^{\Lambda} \neq d \chi_{p-1}^{\Lambda}, \quad \Lambda=1, \ldots, b_{p},
$$

and $b_{p+1}$ closed but not exact $(p+1)$-forms which provide the basis of $\mathbb{H}^{p+1}\left(M^{2 p+2}\right)$,

$$
d \Omega_{p+1}^{L}=0, \quad \Omega_{p+1}^{L}(x) \neq d \chi_{p}^{L}, \quad L=1, \ldots, b_{p+1} .
$$

These latter enter the general solution of the Bianchi identities $d H_{p+1}^{I}=0$,

$$
H_{p+1}^{I}=d B_{p}^{I}+k_{L}^{I} \Omega_{p+1}^{L}
$$

with constant $k_{L}^{I}$ 's.

The PST action (5.8) makes sense in a topologically nontrivial spacetime allowing for the existence of a nowhere vanishing vector field; it can be written for the generalized field strength (5.19). Varying this action within a fixed topological class, $\delta H_{p+1}=d \delta B_{p}$, one finds the same equations (5.13) (see section 3 for more discussion). However, an equivalent first order representation of these equations, which can be obtained as the general solution of (5.13) with respect to $\mathcal{G}_{p}^{I} \wedge d a$, now contains additional topological contributions.

A straightforward generalization of our approach of section 3 allows to show the following facts.

- The first order form of the PST Lagrangian equations (5.13) can be written in the form

$$
\mathcal{G}_{p}^{I} \wedge d a=-d \phi_{p-1}^{I} \wedge d a+\check{\omega}_{p}^{I} \wedge d a,
$$


where $\check{\omega}_{p}^{I}$ are nontrivial solutions of

$$
d \check{\omega}_{p}^{I}=\check{\omega}_{p}^{(1) I} \wedge d a, \quad d \check{\omega}_{p}^{(1) I}=\check{\omega}_{p}^{(2) I} \wedge d a, \quad \ldots, \quad d \check{\omega}_{p}^{(n) I}=\check{\omega}_{p}^{(n+1) I} \wedge d a
$$

In the configuration with $d a=d t$ the above conditions imply that the forms $\check{\omega}_{p}^{I}$ are 'spatially closed' but not 'spatially exact', i.e. obey $d^{(-)} \check{\omega}_{p}^{I}=0$ and $\check{\omega}_{p}^{I} \neq d^{(-)} \chi_{p-1}^{I}$ where $d^{(-)}=d \vec{x} \vec{\partial}$ and $\vec{x}$ are coordinate on the slice $M_{t}^{2 p+1}$ of $M^{2 p+2}$.

- In a spacetime with $b_{p} \neq 0$ a solution of (5.21) is given by $\check{\omega}_{p}^{I}=l_{\Lambda}^{I}(a(x)) \omega_{p}^{\Lambda}$ were $\omega_{p}^{\Lambda}$ are $b_{p} p$-forms forming the basis of $\mathbb{H}^{p}\left(M^{2 p+2}\right),(5.17)$, and $l_{\Lambda}^{I}$ are arbitrary functions of one variables. In the above solution this is taken to be the PST scalar.

- At least for particular cases of $M^{2 p+2}$ the above solution is general (a particular example is $\mathbb{R} \otimes M^{2 p+1}$ with $d a$ co-tangent to $\mathbb{R}$ ) so that

$$
\mathcal{G}_{p}^{I} \wedge d a=-d \phi_{p-1}^{I} \wedge d a+l_{\Lambda}^{I}(a(x)) \omega_{p}^{\Lambda} \wedge d a(x) .
$$

with arbitrary $l_{\Lambda}^{I}(a(x))$. In this case it becomes especially transparent that the value of $b_{p}$ (rather than of $b_{p+1}$ ) is relevant when considering the PST action for $B_{p}$ in $M^{2 p+2}$.

- The complete set of symmetries of the PST action is described by (to simplify equations in this item we omit the superindex ${ }^{I}$ )

$$
\delta B_{p}=d \alpha_{p-1}+\varphi_{p-1} \wedge d a+\delta a \mathcal{G}_{p}+\check{\varphi}_{p}, \quad d \check{\varphi}_{p}=\check{\varphi}_{p}^{(1)} \wedge d a
$$

with

$$
d \check{\varphi}_{p}=\check{\varphi}_{p}^{(1)} \wedge d a, \quad d \check{\varphi}_{p}^{(1)}=\check{\varphi}_{p}^{(2)} \wedge d a, \quad \ldots, \quad d \check{\varphi}_{p}^{(n)}=\check{\varphi}_{p}^{(n+1)} \wedge d a, \quad \ldots
$$

At least in the particular cases (see above) this can be written as

$$
\delta B_{p}=d \alpha_{p-1}+\varphi_{p-1} \wedge d a+\delta a \mathcal{G}_{p}+\omega_{p}^{\Lambda} f_{\Lambda}(a(x))
$$

with $b_{p}$ arbitrary functions of one variable $f_{\Lambda}(a)$. These parametrize the semi-local symmetry. In generic case the parameters of this semi-local symmetry of the PST action are hidden inside of $p$-form $\check{\varphi}_{p}$ which obey the (infinite chain of) equations (5.24).

- The r.h.s. of the first order form of the Lagrangian PST equations, eqs. (5.20) or (5.22), can be removed by the standard PST gauge symmetries, described by the second and the third terms in (5.23) or (5.25), and by the semi-local symmetry.

- Thus if the semi-local symmetry is gauge symmetry, the first order form of the PST Lagrangian equation, (5.20) or (5.22), is gauge equivalent to $\mathcal{G}_{p}^{I}=0$, eq. (5.14), which in its turn is equivalent to the twisted anti-self-duality equation

$$
\mathcal{H}_{p+1}^{I}:=H_{p+1}^{I}+\Omega_{I J} * H_{p+1}^{J}=0
$$

for the (field strengths of the) potentials which enter the action. 
- This is the case for the da-timelike branch of the PST system, and for the HT action which is obtained from this by fixing the gauge $d a=d t$.

- For the other, $d a$-spacelike branch of the PST system, and for the PS action which is obtained from this by fixing the gauge (say) $d a=d x^{5}$, the best what one can obtain is the twisted anti-self-duality equation $\tilde{\mathcal{H}}_{p+1}^{I}:=\tilde{H}_{p+1}^{I}+\Omega_{I J} * \tilde{H}_{p+1}^{I}=0$ for redefined field strength, $\tilde{H}_{p+1}^{I}=H_{p+1}^{I}-l_{\Lambda}^{I}(a(x)) \omega_{p}^{\Lambda} \wedge d a$ in the particular cases (see (5.22)) and $\tilde{H}_{p+1}^{I}=H_{p+1}^{I}-\check{\omega}_{p}^{I} \wedge d a$ in generic spacetime (see (5.21) and (5.20)). As far as $d a$ can be considered as exact form, this can be interpreted as field strength of redefined $p$-form potential, $\tilde{B}_{p}^{I}=B_{p}^{I}-\tilde{f}_{\Lambda}^{I}(a(x)) \omega_{p}^{\Lambda}$ in the particular cases and $\tilde{B}_{p}^{I}=B_{p}^{I}-\check{\beta}_{p}$ with $\check{\beta}_{p}=\check{\omega}_{p} \wedge d a$ in general. Although this redefinition is given by the semi-local symmetry transformation, this is not a gauge symmetry of the $d a$-spacelike branch of the PST system, nor of the PS action, so that its parameters should be considered as additional degrees of freedom of the dynamical system.

To resume, the PST formalism is consistent and can be used to obtain the (twisted anti-)self-duality equations for $p$-form gauge potentials also in spacetime $M^{2 p+2}$ of nontrivial topology (admitting a nowhere vanishing vector field). To be more precise, in the spacetime with $b_{p} \neq 0$ and, more generally, in $M^{2 p+2}$ admitting a nontrivial $p$-forms obeying (5.21) this conclusion holds for the da-timelike branch of the dynamical system described by the PST action, as well as for the non-manifestly invariant HT action which can be obtained from that by gauge fixing.

\section{Conclusions}

In this paper we have shown that the Pasti-Sorokin-Tonin (PST) approach $[2,3]$ is consistent and produces the (twisted anti-)self-duality equation as a gauge fixed version of the equations of motion also in spacetime of nontrivial topology.

We have began by the basic example of chiral 2-form gauge field in $\mathrm{D}=6$ spacetime $M^{6}$, which has been elaborated in detail in sections 2 and 3 . This allowed to shorten the presentation of the generic case of chiral $p$-form gauge fields $B_{p}^{I}$ in $\mathrm{D}=2 \mathrm{p}+2$ dimensional spacetime $M^{2 p+2}=M^{1+(2 p+1)}$ of nontrivial topology in section 5 . The intermediate section 4 is devoted to the special case of $D=2$ chiral bosons.

The PST action contains an auxiliary scalar field $a(x)$ which is pure gauge ( Stückelberg field) with respect to a specific gauge symmetry (PST2 gauge symmetry). However, as far as $\frac{1}{\sqrt{|\partial a(x) \partial a(x)|}}$ enters the action and the Lagrangian equations, not all the configurations of $a(x)$ are allowed. We stress that this topological restriction implies the existence of two branches of the dynamical system described by the PST action (PST system): da-timelike branch in which the PST scalar can be gauged to coincide with time coordinate, or better to say $d a=d t$, and $d a$-spacelike branch in which the gauge $d a=d x^{1}$ is accessible.

In the gauge $d a=d t$ the (da-timelike branch of the) PST action reduces to the (non-manifestly Lorentz invariant) Henneaux-Teitelboim (HT) action [6, 32]. In D=2 such an action was discussed in [20] by Floreanini and Jackiw so that we call this FJ action. In the gauge $d a=d x^{1}$ the (da-spacelike branch of the) PST action reduces to another 
non-manifestly Lorentz invariant functional which in $\mathrm{D}=6$ was considered by Perry and Schwarz [7]; we call this PS action while for its $\mathrm{D}=2$ counterpart we also use the name anti-FJ action.

The PST action can be written in any curved spacetime $M^{2 p+2}=M^{1+(2 p+1)}$ provided it allows for the existence of a nowhere vanishing vector field. However, in some case the topology intervenes the process of derivation of (twisted anti)-self duality equations from the Lagrangian equations of the PST action. Namely, this happens if $M^{2 p+2}=M^{1+(2 p+1)}$ allows for the existence of nontrivial $p$-forms $\check{\omega}_{p}$ which obey $d \check{\omega}_{p}=\check{\omega}_{p}^{(1)} \wedge d a(x)$, where $d a(x)$ is an arbitrary nowhere vanishing closed 1-form (which could be exact and identified with the derivative of the PST scalar field) and $\check{\omega}_{p}^{(1)}$ is implicitly defined by the same equation. If $M^{2 p+2}$ allows for the existence of $b_{p}$ linearly independent closed but not exact $p$-forms $\omega_{p}^{\Lambda}\left(\Lambda=1, \ldots, b_{p}\right)$ at least a particular class of such $\check{\omega}_{p}$ is provided by $\sum_{\Lambda=1}^{b_{p}} f_{\Lambda}(a(x)) \omega_{p}^{\Lambda}$, where $f_{\Lambda}(a)$ are arbitrary functions of one variable.

In this case the first order form of the PST Lagrangian equation acquires an additional contribution to its r.h.s. and, on the first glance, are not gauge equivalent to the (twisted anti-)self duality equation. However, a more careful study shows that in such spacetimes the PST action also possesses an additional semi-local symmetry and that the additional terms in the r.h.s. of the first order form of the Lagrangian PST equations can be removed or generated by the transformations of this semi-local symmetry. Furthermore, we have shown that for the da-timelike branch of the PST system this semi-local symmetry is a gauge symmetry and, hence the additional terms in right hand side can be gauged away reducing the Lagrangian equations to the (twisted-anti-)self duality equations.

In the other da-spacelike branch of the PST system the semi-local symmetry is an infinite dimensional rigid symmetry, similar to the conformal symmetry in $2 \mathrm{~d}$, which cannot be used to remove degrees of freedom. As a result, although the Lagrangian PST equations in this branch can be written as (twisted-anti-)self duality equations for a redefined potential, and the redefinition can be identified with semi-local symmetry transformations, the parameters of these should be considered as parameters of the general solution of the equations of motion and thus as additional degrees of freedom making the content of the model different from just chiral boson(s).

As we have commented in the main text, it is tempting, following [33], to speculate on a hypothetical possibility to improve the situation with da-spacelike branch of the PST system by developing an alternative canonical formalism which uses one of the spacial coordinate instead of time. However, if one would like to deal with two branches of the PST system simultaneously, one should use the same formalism for both, so that our problem remains for one of two branches. This provided us with an additional reason to keep in this paper a more conservative point of view and to stay within the standard canonical formalism.

Thus, curiously enough, the topology makes difference between $d a$-timelike and $d a$ spacelike branches of the PST system making the first preferable as its equations of motion are gauge equivalent to the (twisted-anti-)self duality equations and, hence, the field content in this branch is given by one (or several) chiral boson(s). 
An important problem is to understand the implications of our results for quantum theory of D-dimensional chiral bosons.

Another interesting issue is the influence of the spacetime topology on the generalized PST approach of $[8,9]$ with several PST scalars: $a^{r}=\left(a^{1}, \ldots, a^{q}\right)$ with $q>1(q=3$ in $[8,9])$. Instead of $\frac{1}{\sqrt{|\partial a(x) \partial a(x)|}}$ the generalized PST action and equations of motion would include the inverse $Y_{r s}^{-1}$ of the matrix $Y^{r s}=g^{\mu \nu}(x) \partial_{\mu} a(x)^{r} \partial_{\nu} a^{s}(x)$. Hence the requirement for spacetime manifold to have a nowhere vanishing vector field will be replaced in this case by the requirement of a nowhere singular $q \times q$ matrix $Y^{r s}=g^{\mu \nu}(x) \partial_{\mu} a(x)^{r} \partial_{\nu} a(x), \operatorname{det} Y^{r s} \neq$ 0 , or, equivalently, of the nowhere singular rank q projector $P_{\mu}^{\nu}=\partial_{\mu} a(x)^{r} Y_{r s}^{-1} \partial^{\nu} a^{s}(x)$. (This can be formulated as requirement of the existence of nowhere singular q-plane field). We hope to address this problem in near future.

\section{Acknowledgments}

The author is grateful to Dima Sorokin for collaboration at early stages of this project, numerous useful discussions and comments on final version of the manuscript. He thanks Marc Henneaux for useful conversation. This work was supported in part by research grant FPA2012-35043-C02-01 from MINECO of Spain, by the Basque Government research group grant ITT559-10, and by UPV/EHU under the program UFI 11/55.

\section{A On first order form of the Lagrangian equations of the PST system in flat spacetime}

Here we present some details on the derivation of the first order form (2.23) of the Lagrangian equations (2.21) which follow from the PST action (2.11) in topologically trivial $6 \mathrm{D}$ spacetime.

At first glance, it seems that one can solve (2.21) by a more general expression

$$
\mathcal{G}_{2} \wedge d a=\phi_{2} \wedge d a, \quad d \phi_{2}=\phi_{2}^{(1)} \wedge d a
$$

where $d \phi_{2}^{(1)}=\phi_{2}^{(2)} \wedge d a$ etc. However, we will see that in the case of topologically trivial spacetime this does not go beyond the solution (2.23).

For simplicity, let us discuss the case $d a=d t$, when the PST action reduces to the HT action. Let us define the splitting $\phi_{2}=\phi_{2}^{(-)}+i_{0} \phi_{2} \wedge d t, d=d^{(-)}+d t \partial_{t}$ etc.. Then eq. (A.1) can be written in the form

$$
\mathcal{G}_{2} \wedge d t=\phi_{2}^{(-)} \wedge d t
$$

where $d \phi_{2}^{(-)}=\phi_{2}^{(1)(-)} \wedge d t$. This last equation can be equivalently written as $d^{(-)} \phi_{2}^{(-)}=0$. As far as we are in topologically trivial spacetime, its spacial part is also topologically trivial so that $d^{(-)} \phi_{2}^{(-)}=0$ is solved by $\phi_{2}^{(-)}=d^{(-)} \phi_{1}^{(-)}$. This can be equivalently written as $\phi_{2}^{(-)}=d \phi_{1}-\left(\partial_{t} \phi_{1}^{(-)}-d^{(-)} i_{0} \phi_{1}\right) \wedge d t$. Clearly, only the first term contributes to the r.h.s. of (A.2) which, hence, can be equivalently written in the form of eq. (2.23),

$$
\mathcal{G}_{2} \wedge d t=d \phi_{1} \wedge d t=-d\left(\phi_{1} \wedge d t\right) .
$$




\section{B Noether currents and Noether charges in a $6 \mathrm{~d}$ theory of 2-form gauge potential}

The variation of the $6 \mathrm{~d}$ action $\int \mathcal{L}_{6}$ for the 2 -form potential $B_{2}$ can be written as

$$
\int_{M^{6}} \delta \mathcal{L}_{6}=\int_{M^{6}}\left(\frac{\delta \mathcal{L}_{6}}{\delta B_{2}} \wedge \delta B_{2}+\frac{\delta \mathcal{L}_{6}}{\delta H_{3}} \wedge d \delta B_{2}\right)=\int_{M^{6}} \mathcal{E}_{4} \wedge \delta B_{2}+\int_{M^{6}} d\left(\frac{\delta \mathcal{L}_{6}}{\delta H_{3}} \wedge \delta B_{2}\right),
$$

where

$$
\mathcal{E}_{4}=\frac{\delta \mathcal{L}_{6}}{\delta B_{2}}-d \frac{\delta \mathcal{L}_{6}}{\delta H_{3}}
$$

is the 1.h.s. of the Lagrangian equations of motion. A transformations of the 2-form potential

$$
\delta_{\varphi} B_{2}=R_{2 \mathcal{A}} \varphi^{\mathcal{A}}
$$

with constant parameters $\varphi^{\mathcal{A}}$ and field dependent 2-forms $R_{2 \mathcal{A}}=R_{2 \mathcal{A}}\left(B_{2}\right)$ is a symmetry if $\delta_{\varphi} \mathcal{L}_{6}=\varphi^{\mathcal{A}} d K_{5 \mathcal{A}}$. As, on the other hand, eq. (B.1) implies $\delta_{\varphi} \mathcal{L}_{6}=\mathcal{E}_{4} \wedge R_{2 \mathcal{A}} \varphi^{\mathcal{A}}+$ $d\left(\frac{\delta \mathcal{L}_{6}}{\delta H_{3}} \wedge R_{2 \mathcal{A}}\right)$, we see that the 5 -form

$$
* J_{1 \mathcal{A}}=\frac{\delta \mathcal{L}_{6}}{\delta H_{3}} \wedge R_{2 \mathcal{A}}-K_{5 \mathcal{A}}
$$

is closed on the mass shell,

$$
d * J_{1 \mathcal{A}}=0 \quad \text { when } \quad \mathcal{E}_{4}=0 .
$$

The Noether current $J_{\mathcal{A}}^{\mu}$ can be defined by

$$
* J_{1 \mathcal{A}}=: \frac{1}{5 !} d x^{\mu_{5}} \wedge \ldots \wedge d x^{\mu_{1}} \epsilon_{\mu_{1} \ldots \mu_{5} \mu} J_{\mathcal{A}}^{\mu}
$$

and eq. (B.4) is equivalent to its conservation,

$$
\partial_{\mu} J_{\mathcal{A}}^{\mu}=0 \quad \text { when } \quad \mathcal{E}_{4}=0 .
$$

For a gauge symmetry the 5 -form $((D-1)$-form) dual to the Noether current is not only closed, but exact on the mass shell so that the corresponding Noether charge vanishes identically.

As an example let us consider the standard 2-form gauge symmetry $\delta B_{2}=d \alpha_{1}$ of the action with Lagrangian form dependent on $B_{2}$ through $H_{3}=d B_{2}$ only. In this case the equations of motion read $d\left(\frac{\delta \mathcal{L}_{6}}{\delta H_{3}}\right)=0$. Rewriting the gauge transformations in the form of $\delta B_{2}=d x^{\mu} \wedge d x^{\nu} \sum_{n=0}^{\infty} \frac{1}{n !} x^{\rho_{n}} \ldots x^{\rho_{1}} \partial_{\rho_{1}} \ldots \partial_{\rho_{1}} \partial_{[\nu} \alpha_{\mu]}$ and identifying the constant parameters as $\left\{\varphi^{\mathcal{A}}\right\}=\left\{\frac{1}{n !} \partial_{\rho_{1}} \ldots \partial_{\rho_{1}} \partial_{[\nu} \alpha_{\mu]}\right\}$, we find the 5 -forms dual to Noether currents

$$
* J_{1}^{\nu_{1} \nu_{2} \rho_{1} \ldots \rho_{n}}=\frac{\delta \mathcal{L}_{6}}{\delta H_{3}} \wedge\left(d x^{\nu_{1}} \wedge d x^{\nu_{2}} x^{\rho_{1}} \ldots x^{\rho_{n}}\right)_{\square} \ldots \ldots .
$$


The graphical subscript $\stackrel{\square}{\square} \ldots . . . . . \square$ in this expression indicates that one should take only one irreducible part of the tensorial 2-form in the brackets, that corresponding to the Young diagram represented by the subscript. ${ }^{11}$ This extraction of one irreducible part allows to conclude that $\left(d x^{\nu_{1}} \wedge d x^{\nu_{2}} x^{\rho_{1}} \ldots x^{\rho_{n}}\right)_{\square \square \ldots . . . \square}=\propto d\left(d x^{\left[\nu_{1}\right.} x^{\left.\nu_{2}\right]} x^{\rho_{1}} \ldots x^{\rho_{n}}\right)_{\square \square \ldots . . .}$ and, thus, that, on the mass shell, the 5-form dual to Noether current is exact

$$
* J_{1}^{\nu_{1} \nu_{2} \rho_{1} \ldots \rho_{n}}=\propto d\left(\frac{\delta \mathcal{L}_{6}}{\delta H_{3}} \wedge\left(d x^{\left[\nu_{1}\right.} x^{\left.\nu_{2}\right]} x^{\rho_{1}} \ldots x^{\rho_{n}}\right)_{\square \square \ldots . . .}\right) .
$$

This is tantamount to saying that the Noether current is given by the divergence of an antisymmetric tensor, $J^{\mu \nu_{1} \nu_{2}} \rho_{1} \ldots \rho_{n}=\partial_{\mu^{\prime}}(\ldots)_{1}^{\mu^{\prime} \mu \nu_{1} \nu_{2} \rho_{1} \ldots \rho_{n}}$, so that the Noether charge vanishes, $Q^{\mu \nu_{1} \nu_{2} \rho_{1} \ldots \rho_{n}}=\int d^{5} x J^{0 \nu_{1} \nu_{2} \rho_{1} \ldots \rho_{n}}=\int d^{5} x \partial_{i}(\ldots)_{1}^{i 0 \nu_{1} \nu_{2} \rho_{1} \ldots \rho_{n}}=0$ (we do not consider here the possible boundary contributions).

As a second example, we can consider the PST1 gauge symmetry, or better its counterpart for the Henneaux-Teitelboim action $\delta B_{2}=\phi_{1} \wedge d t=-d t \wedge d x^{i} \phi_{i}(t, \vec{x})$, for which the constant parameters are $-\frac{1}{n !} \partial_{\mu_{1}} \ldots \partial_{\mu_{n}} \phi_{i}(0, \overrightarrow{0})$ and the formal expression for the 5 -forms dual to the Noether currents read

$$
* J_{1}^{i \mu_{1} \ldots \mu_{n}}=\frac{\delta \mathcal{L}_{6}}{\delta H_{3}} \wedge d t \wedge d x^{i} x^{\mu_{1}} \ldots x^{\mu_{n}} .
$$

However, for the Henneaux-Teitelboim action which have the above described symmetry, $\frac{\delta \mathcal{L}_{6}}{\delta H_{3}}=\mathcal{G}_{2} \wedge d t$ so that the Noether current vanishes identically, $* J_{1}^{i \mu_{1} \ldots \mu_{n}} \equiv 0$.

The same conclusion follows for the $\delta B_{2}=\phi_{1} \wedge d x^{5}$ of the Perry-Schwarz action, confirming that this is also a gauge symmetry characterized by vanishing Noether current.

Open Access. This article is distributed under the terms of the Creative Commons Attribution License (CC-BY 4.0), which permits any use, distribution and reproduction in any medium, provided the original author(s) and source are credited.

\section{References}

[1] P. Pasti, D.P. Sorokin and M. Tonin, Duality symmetric actions with manifest space-time symmetries, Phys. Rev. D 52 (1995) 4277 [hep-th/9506109] [INSPIRE].

[2] P. Pasti, D.P. Sorokin and M. Tonin, On Lorentz invariant actions for chiral $p$ forms, Phys. Rev. D 55 (1997) 6292 [hep-th/9611100] [INSPIRE].

[3] P. Pasti, D.P. Sorokin and M. Tonin, Covariant action for a D=11 five-brane with the chiral field, Phys. Lett. B 398 (1997) 41 [hep-th/9701037] [INSPIRE].

[4] I.A. Bandos et al., Covariant action for the superfive-brane of M-theory, Phys. Rev. Lett. 78 (1997) 4332 [hep-th/9701149] [INSPIRE].

[5] M. Aganagic, J. Park, C. Popescu and J.H. Schwarz, World volume action of the M-theory five-brane, Nucl. Phys. B 496 (1997) 191 [hep-th/9701166] [INSPIRE].

\footnotetext{
${ }^{11}$ The tensorial 2-form $d x^{\mu} \wedge d x^{\nu} x^{\rho_{n}} \ldots x^{\rho_{1}}$ carries reducible representation of the Lorentz group $\stackrel{\square}{\square}$

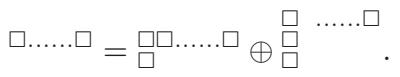


[6] M. Henneaux and C. Teitelboim, Dynamics of Chiral (Selfdual) P Forms, Phys. Lett. B 206 (1988) 650 [INSPIRE].

[7] M. Perry and J.H. Schwarz, Interacting chiral gauge fields in six-dimensions and Born-Infeld theory, Nucl. Phys. B 489 (1997) 47 [hep-th/9611065] [INSPIRE].

[8] P. Pasti, I. Samsonov, D. Sorokin and M. Tonin, BLG-motivated Lagrangian formulation for the chiral two-form gauge field in D=6 and M5-branes, Phys. Rev. D 80 (2009) 086008 [arXiv:0907.4596] [INSPIRE].

[9] S.-L. Ko, D. Sorokin and P. Vanichchapongjaroen, The M5-brane action revisited, JHEP 11 (2013) 072 [arXiv: 1308.2231] [INSPIRE].

[10] D. Belov and G.W. Moore, Holographic Action for the Self-Dual Field, hep-th/0605038 [INSPIRE].

[11] P.-M. Ho and Y. Matsuo, M5 from M2, JHEP 06 (2008) 105 [arXiv:0804.3629] [INSPIRE].

[12] W.-M. Chen and P.-M. Ho, Lagrangian Formulations of Self-dual Gauge Theories in Diverse Dimensions, Nucl. Phys. B 837 (2010) 1 [arXiv:1001.3608] [INSPIRE].

[13] G. Dall'Agata, K. Lechner and M. Tonin, Covariant actions for $N=1, D=6$ supergravity theories with chiral bosons, Nucl. Phys. B 512 (1998) 179 [hep-th/9710127] [INSPIRE].

[14] I.A. Bandos, N. Berkovits and D.P. Sorokin, Duality symmetric eleven-dimensional supergravity and its coupling to M-branes, Nucl. Phys. B 522 (1998) 214 [hep-th/9711055] [INSPIRE].

[15] G. Dall'Agata, K. Lechner and D.P. Sorokin, Covariant actions for the bosonic sector of $D=10$ IIB supergravity, Class. Quant. Grav. 14 (1997) L195 [hep-th/9707044] [INSPIRE].

[16] G. Dall'Agata, K. Lechner and M. Tonin, $D=10, N=I I B$ supergravity: Lorentz invariant actions and duality, JHEP 07 (1998) 017 [hep-th/9806140] [INSPIRE].

[17] I. Bandos, H. Samtleben and D. Sorokin, Duality-symmetric actions for non-Abelian tensor fields, Phys. Rev. D 88 (2013) 025024 [arXiv: 1305.1304] [INSPIRE].

[18] X. Bekaert and M. Henneaux, Comments on chiral p forms, Int. J. Theor. Phys. 38 (1999) 1161 [hep-th/9806062] [INSPIRE].

[19] A. Maznytsia, C.R. Preitschopf and D.P. Sorokin, Duality of selfdual actions, Nucl. Phys. B 539 (1999) 438 [hep-th/9805110] [INSPIRE].

[20] R. Floreanini and R. Jackiw, Selfdual Fields as Charge Density Solitons, Phys. Rev. Lett. 59 (1987) 1873 [INSPIRE].

[21] C. Nash and S. Sen, Topology And Geometry For Physicists, Academic Press INC LTD, London U.K. (1983).

[22] N.J. Hitchin, The geometry of three-forms in six and seven dimensions, math/0010054 [INSPIRE].

[23] N.J. Hitchin, The Geometry of three forms in six-dimensions, J. Diff. Geom. 55 (2000) 547 [INSPIRE].

[24] N. Berkovits, Manifest electromagnetic duality in closed superstring field theory, Phys. Lett. B 388 (1996) 743 [hep-th/9607070] [INSPIRE].

[25] I. Bengtsson and A. Kleppe, On chiral p forms, Int. J. Mod. Phys. A 12 (1997) 3397 [hep-th/9609102] [INSPIRE]. 
[26] I. Giannakis and V.P. Nair, Symplectic structures and selfdual fields in (4k+2)-dimensions, Phys. Lett. B 409 (1997) 145 [hep-th/9702024] [INSPIRE].

[27] E. Bergshoeff, R. Kallosh, T. Ortín, D. Roest and A. Van Proeyen, New formulations of $D=10$ supersymmetry and D8 - O8 domain walls, Class. Quant. Grav. 18 (2001) 3359 [hep-th/0103233] [INSPIRE].

[28] M. Cederwall, B.E.W. Nilsson and P. Sundell, An Action for the superfive-brane in D $=11$ supergravity, JHEP 04 (1998) 007 [hep-th/9712059] [INSPIRE].

[29] D. Zwanziger, Local Lagrangian quantum field theory of electric and magnetic charges, Phys. Rev. D 3 (1971) 880 [INSPIRE].

[30] A. Sevrin and D.C. Thompson, A Note on Supersymmetric Chiral Bosons, JHEP 07 (2013) 086 [arXiv: 1305.4848] [INSPIRE].

[31] M. Henneaux and C. Teitelboim, Consistent quantum mechanics of chiral p-forms, In Santiago 1987, Proceedings, Quantum mechanics of fundamental systems V2, 113-152 (1987).

[32] M. Henneaux and C. Teitelboim, Quantization of Gauge Systems, Princeton University Press, Princeton U.S.A. (1994).

[33] C.M. Hull and N. Lambert, Emergent Time and the M5-Brane, JHEP 06 (2014) 016 [arXiv: 1403.4532] [INSPIRE].

[34] N. Berkovits, Super Poincaré covariant quantization of the superstring, JHEP 04 (2000) 018 [hep-th/0001035] [INSPIRE].

[35] N. Berkovits, Multiloop amplitudes and vanishing theorems using the pure spinor formalism for the superstring, JHEP 09 (2004) 047 [hep-th/0406055] [INSPIRE].

[36] E. Witten, Five-brane effective action in M-theory, J. Geom. Phys. 22 (1997) 103 [hep-th/9610234] [INSPIRE].

[37] W.-M. Chen, P.-M. Ho, H.-c. Kao, F.S. Khoo and Y. Matsuo, Partition function of a chiral boson on a 2-torus from the Floreanini-Jackiw Lagrangian, PTEP 2014 (2014) 033 B02 [arXiv: 1307.2172] [INSPIRE]. 